\title{
Regulation of glucose and lipid metabolism by dietary carbohydrate levels and lipid sources in gilthead sea bream juveniles
}

\author{
Carolina Castro $^{1,2 *}$, Geneviève Corraze ${ }^{3}$, Alexandre Firmino-Diógenes ${ }^{1,2}$, Laurence Larroquet ${ }^{3}$, \\ Stéphane Panserat ${ }^{3}$ and Aires Oliva-Teles ${ }^{1,2}$ \\ ${ }^{1}$ Departamento de Biologia, Faculdade de Ciências, Universidade do Porto, Rua do Campo Alegre, Edifício FC4, 4169-007 \\ Porto, Portugal \\ ${ }^{2}$ CIMAR/CIIMAR - Centro Interdisciplinar de Investigação Marinha e Ambiental, Universidade do Porto, Rua dos Bragas 289, \\ 4050-123 Porto, Portugal \\ ${ }^{3}$ INRA, UR1067 Nutrition Metabolism Aquaculture, F-64310 Saint-Pée-sur-Nivelle, France
}

(Submitted 20 December 2015 - Final revision received 16 March 2016 - Accepted 23 March 2016 - First published online 10 May 2016)

\section{Abstract}

The long-term effects on growth performance, body composition, plasma metabolites, liver and intestine glucose and lipid metabolism were assessed in gilthead sea bream juveniles fed diets without carbohydrates (CH-) or carbohydrate-enriched (20\% gelatinised starch, $\mathrm{CH}+$ ) combined with two lipid sources (fish oil; or vegetable oil (VO)). No differences in growth performance among treatments were observed. Carbohydrate intake was associated with increased hepatic transcripts of glucokinase but not of 6-phosphofructokinase. Expression of phosphoenolpyruvate carboxykinase was down-regulated by carbohydrate intake, whereas, unexpectedly, glucose 6-phosphatase was upregulated. Lipogenic enzyme activities (glucose-6-phosphate dehydrogenase, malic enzyme, fatty acid synthase) and $\Delta 6$ fatty acyl desaturase (FADS2) transcripts were increased in liver of fish fed $\mathrm{CH}+$ diets, supporting an enhanced potential for lipogenesis and long-chain PUFA (LC-PUFA) biosynthesis. Despite the lower hepatic cholesterol content in $\mathrm{CH}+$ groups, no influence on the expression of genes related to cholesterol efflux (ATP-binding cassette G5) and biosynthesis (lanosterol $14 \alpha$-demethylase, cytochrome P450 51 cytochrome P450 51 (CYP51A1); 7-dehydrocholesterol reductase) was recorded at the hepatic level. At the intestinal level, however, induction of CYP51A1 transcripts by carbohydrate intake was recorded. Dietary VO led to decreased plasma phospholipid and cholesterol concentrations but not on the transcripts of proteins involved in phospholipid biosynthesis (glycerol-3-phosphate acyltransferase) and cholesterol metabolism at intestinal and hepatic levels. Hepatic and muscular fatty acid profiles reflected that of diets, despite the up-regulation of $F A D S 2$ transcripts. Overall, this study demonstrated that dietary carbohydrates mainly affected carbohydrate metabolism, lipogenesis and LC-PUFA biosynthesis, whereas effects of dietary lipid source were mostly related with tissue fatty acid composition, plasma phospholipid and cholesterol concentrations, and LC-PUFA biosynthesis regulation. Interactions between dietary macronutrients induced modifications in tissue lipid and glycogen content.

\section{Key words: Carbohydrate content: Cholesterol: Fatty acid bioconversion: Lipid sources: Nutrient metabolism}

Besides being a source of high-quality protein and essential micronutrients for humans, fish are unique sources of $n-3$ longchain PUFA (LC-PUFA), namely EPA (20:5n-3) and DHA (22:6n-3), which were proven to be beneficial for human health $^{(1)}$. Driven by the crescent awareness of the health beneficial effects of $n$ - 3 LC-PUFA in a range of human pathologies (including CVD, inflammatory and neurological diseases) the global fish consumption is rising $^{(1)}$ and an increasing proportion of this fish is now being supplied by aquaculture ${ }^{(2)}$.

Fishmeal (FM) and fish oil (FO) have been widely used as main ingredients in aquafeeds for carnivorous fish species. However, environmental sustainability and economical issues, related to the limited availability of fisheries resources and escalating costs, forced the aquafeed industry to search for alternative and eco-friendly ingredients such as plant feedstuffs and vegetable oils (VO). However, the unbalanced amino acid and fatty acid (FA) profiles, relatively high amounts of carbohydrates and the presence of antinutritional factors may limit their use in aquafeeds, especially for carnivorous fish species that are metabolically adapted to diets rich in LC-PUFA and almost devoid of carbohydrates ${ }^{(1,3-5)}$.

It is well known that modifications of dietary macronutrients (e.g. lipid source, carbohydrates, etc.) may have marked effects on tissue FA composition and lipid deposition of fish ${ }^{(6-9)}$.

Abbreviations: CYP51A1, lanosterol $14 \alpha$-demethylase, cytochrome P450 51; elovl5, elongase 5; FA, fatty acid; FAS, fatty acid synthase; FO, fish oil; G6Pase, glucose 6-phosphatase; G6PD, glucose-6-phosphate dehydrogenase; LC-PUFA, long-chain PUFA; ME, malic enzyme; PL, phospholipids; VO, vegetable oil.

* Corresponding author: C. Castro, email carolinacastro23@gmail.com 
For instance, replacement of $\mathrm{FO}$ by $\mathrm{VO}$ generally leads to a decrease of $n$ - 3 LC-PUFA and an increase of 18C PUFA precursors, linoleic acid (18:2n-6) and $\alpha$-linolenic acid $(18: 3 n-3)$, in the fillet ${ }^{(7,9-11)}$. Increased tissue lipid deposition with dietary incorporation of $\mathrm{VO}^{(6,8,9)}$ or carbohydrates ${ }^{(7,12)}$ was also reported. Such effects may occur because of different mechanisms that are recognised to regulate the quantity and quality of the fish lipid depots, such as modification of lipogenesis, $\beta$-oxidation, tissue lipid uptake and transport or FA desaturation and elongation processes ${ }^{(13,14)}$.

Although not always consistent, several studies in fish reported that dietary lipid source and carbohydrates regulate gene expression, key transcription factors and/or activity of enzymes involved in lipogenesis, $\beta$-oxidation and lipid uptake. Accordingly, it was observed that replacing dietary $\mathrm{FO}$ by $\mathrm{VO}$ decreased $^{(15,16)}$ or increased $^{(17-19)}$ gene expression or activity of lipogenic enzymes (such as glucose-6-phosphate dehydrogenase (G6PD), malic enzyme (ME), fatty acid synthase (FAS)). Similarly, dietary VO was reported to regulate gene expression or activities of lipolytic enzymes either positively ${ }^{(20,21)}$ or negatively ${ }^{(19,20,22,23)}$ (including carnitine palmitoyltransferase (CPT) I and II, lipoprotein lipase) in a tissue-specific manner. Dietary carbohydrate or glucose administration was also reported to enhance lipogenesis ${ }^{(24)}$. However, regarding $\beta$-oxidation, data are contradictory, as either stimulation ${ }^{(25)}$ or inhibition ${ }^{(26)}$ effects were reported. Regarding LC-PUFA biosynthesis, strong induction of desaturase (fatty acyl desaturase (FAD) $\Delta 5$ and $\Delta 6$ desaturases, FADS1 and $F A D S 2$, respectively) and elongase expression by VO administration was reported in freshwater fish and salmonids ${ }^{(10,27)}$, but in marine fish such induction of gene expression is not so clear ${ }^{(11,28-30)}$. Desaturases and elongases were also shown to be up-regulated by dietary carbohydrates in salmonids ${ }^{(25,27)}$, but in marine fish such an effect has never been demonstrated ${ }^{(7)}$.

Besides the reported effects on tissue FA composition and lipid deposition of fish, the use of plant feedstuffs and VO can also compromise fish physiological functions and, ultimately, fish health ${ }^{(31,32)}$. For example, there is an increased awareness of potential effects due to reduced dietary phospholipids (PL) or cholesterol in plant feedstuff-based diets. Besides their important roles in membrane structure, cholesterol and PL also have important functional roles. Cholesterol is a precursor of physiologically active compounds such as bile acids, vitamin D, adrenal corticoids and sex hormones, and PL are precursors of eicosanoids, diacylglycerol, inositol phosphates and plateletactivating factors ${ }^{(33,34)}$. In a number of fish species, dietary VO was reported to decrease plasma $\mathrm{PL}^{(7,35,36)}$, cholesterol and LDL-cholesterol ${ }^{(8,35,37)}$, and to modulate the activity or expression of genes involved in PL synthesis ${ }^{(38)}$ and cholesterol synthesis and absorption $^{(7,10,39)}$. Recently, it was also demonstrated that dietary carbohydrates regulate plasma cholesterol and PL concentrations and the transcription of proteins involved in cholesterol metabolism ${ }^{(7)}$.

Therefore, to provide adequate background for successful use of plant feedstuffs in aquafeeds, all aspects related to fish physiological functions and the nutritional quality of the final product must be better understood. For that purpose, we assessed the long-term effect of dietary lipid source, carbohydrate content and interactions between both on growth performance, tissue composition, liver and intestine enzymatic activity and expression of genes related with lipid metabolism (lipogenesis, $\beta$-oxidation, FA bioconversion, cholesterol and PL metabolism) in gilthead sea bream juveniles. In this study, the selection of the relevant genes involved in lipid metabolism was performed taking advantage of the recent advances in the molecular and functional characterisation of a number of new gilthead sea bream sequences related to FA, PL and cholesterol metabolism ${ }^{(40-47)}$. In addition, as an increasing number of studies have reported that glucose metabolism was distinctly regulated by different dietary lipid sources $^{(7,17,48-50)}$, we also investigated the effects of these dietary manipulations on mechanisms involved in glucose utilisation/metabolism (such as glycolysis and gluconeogenesis pathways).

\section{Methods}

\section{Experimental diets}

Four diets differing in carbohydrate content (0 and 20\% gelatinised starch, diets $\mathrm{CH}-$ and $\mathrm{CH}+$, respectively) and lipid source (diets FO or VO) were formulated (Table 1). The increase in carbohydrate content in $\mathrm{CH}+$ diets was achieved by decreasing protein, which was kept well above the requirements of the species ${ }^{(51)}$. The major lipid source of FO diets was cod liver oil. In VO diets, $100 \%$ of the cod liver oil was replaced by a VO blend composed of $20 \%$ rapeseed, $50 \%$ linseed and $30 \%$ palm oils. FM was added as a major dietary protein source to isolate the impacts of dietary $\mathrm{VO}$ and to avoid the interference of dietary plant protein on lipid metabolism, especially on cholesterol metabolism.

\section{Animals, experimental conditions and sampling}

The experiment was directed by trained scientists (following FELASA category $\mathrm{C}$ recommendations) and conducted according to the European Union Directive (2010/63/EU) on the protection of animals for scientific purposes. The study was performed at the Marine Zoological Station, University of Porto, Portugal, in a thermoregulated recirculation water system equipped with twelve fibreglass cylindrical tanks of 300 litres water capacity, and supplied with continuous flow of filtered seawater. After 2 weeks of adaptation to the experimental conditions, twelve groups of twenty-one gilthead sea bream (Sparus aurata) juveniles with an initial body weight of $70 \cdot 8$ (sD 0.03) g were established and the experimental diets were randomly assigned to triplicate groups of these fish.

The growth trial lasted for $81 \mathrm{~d}$, and during this period fish were hand-fed twice a day, $6 \mathrm{~d} /$ week, to apparent visual satiety. At the end of the trial, fish were unfed for $1 \mathrm{~d}$ to empty the gut content and then bulk-weighed after mild anesthesia with $0.3 \mathrm{ml} / 1$ methylethanol. At the beginning and at the end of the growth trial, fifteen fish from the stock population and three fish from each tank were, respectively, sampled, pooled and frozen until whole-body composition analysis. During the trial, salinity averaged 34.7 (SD 0.8) g/l, dissolved $\mathrm{O}_{2}$ was kept near saturation and water temperature was regulated to 24.0 (sD 0.5$)^{\circ} \mathrm{C}$. 
Table 1. Ingredient and chemical composition of the experimental diets

\begin{tabular}{|c|c|c|c|c|}
\hline \multirow{3}{*}{$\begin{array}{l}\text { Lipid source... } \\
\mathrm{CH} . . .\end{array}$} & \multicolumn{4}{|c|}{ Experimental diets } \\
\hline & \multicolumn{2}{|c|}{ FO } & \multicolumn{2}{|c|}{ VO } \\
\hline & $\mathrm{CH}-$ & $\mathrm{CH}+$ & $\mathrm{CH}-$ & $\mathrm{CH}+$ \\
\hline \multicolumn{5}{|l|}{ Ingredients (\% dry weight) } \\
\hline Fishmeal $^{*}$ & $87 \cdot 3$ & $65 \cdot 1$ & $87 \cdot 3$ & $65 \cdot 1$ \\
\hline Starch† & 0 & 20 & 0 & 20 \\
\hline Cod liver oil & $9 \cdot 2$ & 11.4 & 0 & 0 \\
\hline VO§ & 0 & 0 & $9 \cdot 2$ & 11.4 \\
\hline Vitamins $\|$ & 1.5 & 1.5 & 1.5 & 1.5 \\
\hline Minerals & 1.0 & 1.0 & 1.0 & 1.0 \\
\hline Binder** & 1.0 & 1.0 & 1.0 & 1.0 \\
\hline \multicolumn{5}{|c|}{ Proximate analyses (\% DM) } \\
\hline DM & $87 \cdot 0$ & $86 \cdot 8$ & $87 \cdot 2$ & 87.6 \\
\hline Crude protein (CP) & $66 \cdot 3$ & $50 \cdot 3$ & $66 \cdot 3$ & $50 \cdot 4$ \\
\hline Crude fat $(\mathrm{CF})$ & 18.4 & $18 \cdot 4$ & $18 \cdot 2$ & $18 \cdot 3$ \\
\hline Starch & - & $16 \cdot 8$ & - & $18 \cdot 0$ \\
\hline Energy (kJ/g DM) & $22 \cdot 7$ & $23 \cdot 3$ & $23 \cdot 3$ & $22 \cdot 7$ \\
\hline Ash & $14 \cdot 1$ & $11 \cdot 2$ & $14 \cdot 3$ & $11 \cdot 1$ \\
\hline Cholesterol & 0.59 & 0.39 & 0.49 & 0.39 \\
\hline Protein/energy (g/MJ) & $29 \cdot 2$ & $21 \cdot 6$ & 28.5 & $22 \cdot 2$ \\
\hline \multicolumn{5}{|c|}{ 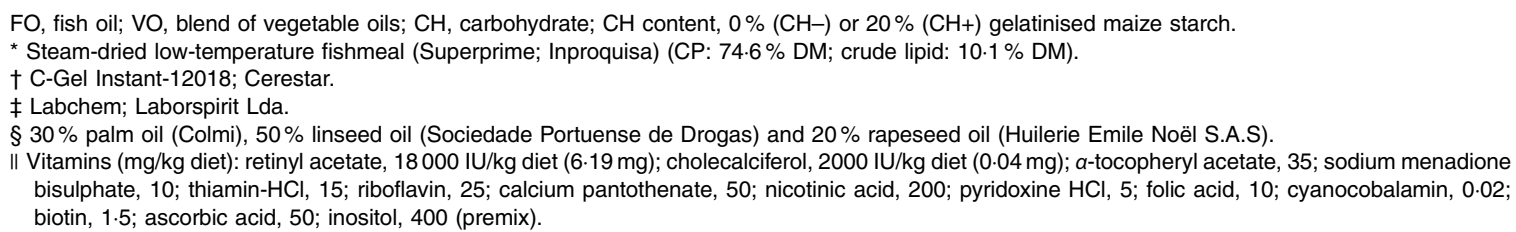 } \\
\hline \multicolumn{5}{|c|}{$\begin{array}{l}\text { II Minerals ( } \mathrm{mg} / \mathrm{kg} \text { diet): cobalt sulphate, } 1.91 \text {; copper sulphate, } 19.6 \text {; iron sulphate, 200; sodium fluoride, } 2.21 \text {; potassium iodide, } 0.78 \text {; magnesium oxide, } \\
830 ; \text { manganese oxide, } 26 \text {; sodiumselenite, } 0.66 \text {; zinc oxide, 37.5; dibasic calcium phosphate, } 5.93 \text { (g/kg diet); potassium chloride, } 1.15 \text { (g/kg diet); } \\
\text { sodium chloride, } 0.40 \text { (g/kg diet) (premix). } \\
\text { ** Aquacube (guar gum, polymethyl carbamide, manioc starch blend, hydrate calcium sulphate) (Agil). }\end{array}$} \\
\hline
\end{tabular}

To eliminate handling stress, after the growth trial fish, continued to be fed for one more week, and then $18 \mathrm{~h}$ after the last meal (the previous day afternoon meal) nine fish from each tank were randomly sampled for blood, liver, intestine and muscle collection. Blood was collected from the caudal vein using heparinised syringes and centrifuged at $2500 \boldsymbol{g}$ for $10 \mathrm{~min}$ and the recovered plasma was kept at $-20^{\circ} \mathrm{C}$ until analysis. Thereafter, fish were killed with a sharp blow to the head, and whole body, viscera and liver were weighed for determination of hepatosomatic (HSI) and viscerosomatic (VSI) indexes. Liver, intestine and muscle sections were frozen in liquid $\mathrm{N}_{2}$ and then stored at $-80^{\circ} \mathrm{C}$ until biochemical, enzymatic and molecular analyses.

\section{Diets, whole fish, liver, muscle and plasma analysis}

Chemical analysis of the diets, whole fish, liver and muscle was conducted according to $\mathrm{AOAC}^{(52)}$ and by following the procedures given below: DM after drying at $105^{\circ} \mathrm{C}$ until constant weight; ash by incineration in a muffle furnace at $450^{\circ} \mathrm{C}$ for $16 \mathrm{~h}$; protein content $(\mathrm{N} \times 6 \cdot 25)$ by the Kjeldahl method after acid digestion using a Kjeltec digestion and distillation unit (models 1015 and 1026, Tecator Systems; Höganäs); and lipid by petroleum diethyl ether extraction (Soxtec HT System; Höganäs). Starch was determined according to Beutler ${ }^{(53)}$ and gross energy by direct combustion in an adiabatic bomb calorimeter (PARR model 6200; Parr Instruments).
Hepatic and muscular glycogen contents were determined as described by Roehrig \& Allred $^{(54)}$, and lipids were determined according to the method of Folch et al. ${ }^{(55)}$. FA methyl esters were prepared by acid transmethylation of total lipids using boron trifluoride in methanol (14\%), as described by Shantha \& Ackman $^{(56)}$, and analysed by GC (Varian 3900; Varian; for details see Castro et al. $\left.{ }^{(48)}\right)$. Total cholesterol in diets, liver and muscle was assayed on total lipid extract by the LiebermannBurchard method ${ }^{(57)}$. Plasma metabolites were analysed using commercial kits from Spinreact: glucose (ref: 1001191), TAG (ref: 1001312), total cholesterol (ref: 1001090) and PL (ref: 1001140).

\section{Enzymatic activity assays}

The activity of key lipogenesis enzymes was determined in the liver (three fish per tank). For that purpose, liver was homogenised (dilution 1:4) in ice-cold buffer (100 mm-Tris- $\mathrm{HCl}$, $0.1 \mathrm{~mm}$-EDTA and $0.1 \%$ triton X-100 (v/v), $\mathrm{pH} 7 \cdot 8)$. All procedures were performed on ice. Homogenates were centrifuged at $30000 \mathrm{~g}$ for $30 \mathrm{~min}$ at $4^{\circ} \mathrm{C}$. After centrifugation, the resultant supernatant was collected and aliquots were stored at $-80^{\circ} \mathrm{C}$ until analysis. All enzyme activities were measured at $37^{\circ} \mathrm{C}$, by monitoring the changes in absorbance of $\mathrm{NADPH}$ at $340 \mathrm{~nm}$ in a Multiskan GO microplate reader (model 5111 9200; Thermo Scientific), using $6.22 \mathrm{~mm} / \mathrm{cm}$ as the millimolar extinction coefficient for NADPH. The optimal 
substrate and protein concentrations for measurement of each enzyme activity were established by preliminary assays. G6PD (EC 1.1.1.49), ME (EC 1.1.1.40) and FAS (EC 2.3.1.38) activities were determined as previously described by Castro et al. ${ }^{(7)}$.

\section{Gene expression}

Analyses of mRNA levels were performed on liver and intestine samples (two fish per tank). Tissues for RNA analyses were homogenised in 2-ml tubes containing Trizol reagent (Invitrogen) using rapid vibration (liver: $2 \times 10 \mathrm{~s}$, with an interval of $10 \mathrm{~s}$, at $5000 \mathrm{rpm}$; intestine: $3 \times 10 \mathrm{~s}$, with $10 \mathrm{~s}$ intervals, at $6500 \mathrm{rpm}$ ) in Precellys ${ }^{\circledR} 24$ (Bertin Technologies). Extraction of total RNA was then performed according to the manufacturer's recommendations. RNA quality and quantity were assessed by gel electrophoresis and spectrophotometry (NanoDrop ND1000; NanoDrop Labtech). Complementary DNA (cDNA) synthesis was performed with $1 \mu \mathrm{g}$ of the resulting total RNA using SuperScript III RNaseH-Reverse Transcriptase kit (Invitrogen) and random primers (Promega). Real-time quantitative PCR (q-PCR) analyses were performed in a total volume of $6 \mu \mathrm{l}$ (detailed information of the reaction mix in Castro et $a l^{(7)}$ ) using LightCycler ${ }^{\circledR} 480$ II apparatus (Roche Diagnostics) to assess the gene expression levels. Primers were either obtained in the literature (Mininni et al. ${ }^{(44)}$; PérezSánchez et al. ${ }^{(45)}$; Sánchez-Gurmaches et al. ${ }^{(46)}$; Enes et al. ${ }^{(59)}$; Diez et $\left.a l .{ }^{(74)}\right)$ or designed from gilthead sea bream-expressed sequence tag sequences available on the SIGENAE database (http://www.sigenae.org) using the Primer3 software ${ }^{(58)}$ (online Supplementary Table S1). For gene targets that had not been previously validated, primers were tested on a pool of cDNA and amplified products were systematically sequenced. The PCR protocol followed the conditions described previously by Castro et al. $^{(7)}$. Each PCR run included duplicates of reverse transcription for each sample and negative controls (RT-free samples, RNA-free samples). PCR run for reference gene included quadruplicates for each sample (duplicates of reverse transcription and PCR amplification, respectively) and negative controls. Quantification of the target gene transcripts in the liver and intestine was done using $\beta$-actin gene expression as reference, as previously used in gilthead sea bream by Pérez-Sánchez et $a l .{ }^{(45)}$, and that was stably expressed in the present study (data not shown). Relative quantification of the target gene transcript with the $\beta$-actin reference gene transcript was performed using the mathematical model described by Pfaffl $^{(60)}$. The relative expression ratio $(R)$ of a target gene was calculated on the basis of real-time PCR efficiency $(E)$ and the threshold cycle $\left(C_{t}\right)$ deviation $(\Delta C T)$ of the unknown sample compared with a control sample and expressed in comparison with the $\beta$-actin reference gene:

$R=[($ Etarget gene $) \Delta C T$ target gene (mean control-mean sample) $]$

$$
/[(\mathrm{E} \beta \text { actin }) \Delta C T \beta \operatorname{actin}(\text { mean control-mean sample })] \text {. }
$$

Efficiency of q-PCR was measured by the slope of a standard curve using serial dilutions of cDNA. The fish fed the FOCH diet was used as the control group.

\section{Statistical analysis}

Data were checked for normality and homogeneity of variances, and they were normalised when appropriate. Statistical evaluation of data was carried out by a $2 \times 2$ factorial arrangement of treatments in a completely randomised experimental design (two-way ANOVA) with carbohydrate content and lipid source as fixed factors. The significance level of 0.05 was used for rejection of the null hypothesis. In cases in which interaction was significant, one-way ANOVA was performed for each factor. All statistical analyses were conducted using the SPSS 22.0 software package (IBM Corp.) for Windows.

\section{Results}

\section{Dietary fatty acid composition}

The four diets presented small differences in the proportions of total SFA, whereas MUFA were higher in FO diets, and $n-3$ an $n$-6 PUFA were higher in VO diets (Table 2). Within MUFA, high levels of oleic acid (18:1n-9) were recorded in VO diets, whereas the opposite occurred for palmitoleic acid (16:1n-7), eicosenoic acid (20:1n-9) and erucic acid (22:1n-9). Among $n-3$ PUFA, VO diets were particularly rich in linolenic acid $(18: 3 n-3)$ and poor in EPA and DHA. The proportion of total $n$-6 PUFA was strongly higher in VO diets mainly because of linoleic acid (18:2n-6) levels.

\section{Growth performance and feed utilisation}

Fish promptly accepted the experimental diets, and no mortality was recorded during the trial. Dietary treatments had no effects on fish growth performance or feed utilisation (Table 3). Feed intake $(\mathrm{g} / \mathrm{kg}$ average body weight per $\mathrm{d})$ was similar among diets. $\mathrm{N}$ retention, expressed per unit weight gain, was not affected by diet composition. However, protein efficiency ratio was higher with the $\mathrm{CH}+$ diets, which had a lower protein content. Dietary carbohydrate intake increased lipid retention per unit weight gain only when fish were fed the VO diet (Table 3). In fish fed $\mathrm{CH}$ - diets, lipid retention per unit weight gain was lower in the VO group. In addition, within the VO group, lipid retention per unit weight gain was lower in fish fed the $\mathrm{CH}-$ diet (carbohydrate and lipid source interaction).

\section{Whole-body, liver and muscle composition}

At the end of the trial, only whole-body lipid and DM contents were affected by dietary treatments. Under a VO-based diet regimen, whole-body lipid content was higher in $\mathrm{CH}+$ than in $\mathrm{CH}-$ groups. In addition, whole-body lipid was lower in VO than in FO groups only when fish were fed no-carbohydrate diets ( $\mathrm{CH}$ - diets) (carbohydrate and lipid source interaction). Whole-body DM content was higher in fish fed the $\mathrm{CH}+$ diets (Table 4).

Higher HSI and VSI were observed in the $\mathrm{CH}+$ groups, but no effect of dietary lipid source was noticed (Table 4). 
Table 2. Fatty acid composition (\% of total fatty acids) of the experimental diets

\begin{tabular}{|c|c|c|c|c|}
\hline \multirow{3}{*}{$\begin{array}{l}\text { Lipid source... } \\
\mathrm{CH} . .\end{array}$} & \multicolumn{4}{|c|}{ Experimental diets } \\
\hline & \multicolumn{2}{|c|}{ FO } & \multicolumn{2}{|c|}{ VO } \\
\hline & $\mathrm{CH}-$ & $\mathrm{CH}+$ & $\mathrm{CH}-$ & $\mathrm{CH}+$ \\
\hline $14: 0$ & 5.8 & 5.9 & 2.5 & $2 \cdot 0$ \\
\hline $15: 0$ & 0.7 & 0.6 & 0.4 & 0.3 \\
\hline $16: 0$ & $18 \cdot 7$ & $17 \cdot 2$ & $21 \cdot 3$ & $21 \cdot 0$ \\
\hline $17: 0$ & 0.5 & 0.4 & 0.4 & 0.3 \\
\hline $18: 0$ & 4.0 & 3.5 & 4.9 & 4.5 \\
\hline $20: 0$ & 0.2 & $0 \cdot 1$ & 0.3 & 0.2 \\
\hline$\sum \mathrm{SFA}$ & 29.9 & $27 \cdot 8$ & 29.9 & $28 \cdot 6$ \\
\hline $16: 1 n-7$ & $7 \cdot 1$ & 7.5 & $2 \cdot 3$ & $1 \cdot 8$ \\
\hline $18: 1 n-9$ & $18 \cdot 3$ & $19 \cdot 1$ & $25 \cdot 4$ & $27 \cdot 6$ \\
\hline $20: 1 n-9$ & 4.9 & $5 \cdot 8$ & 0.9 & 0.7 \\
\hline $22: 1 n-9$ & 3.9 & 4.3 & 0.5 & 0.4 \\
\hline$\sum$ MUFA & 34.4 & $36 \cdot 8$ & 29.2 & 30.5 \\
\hline $18: 2 n-6$ & $2 \cdot 2$ & $2 \cdot 2$ & $9 \cdot 0$ & $10 \cdot 6$ \\
\hline $18: 3 n-6$ & 0.1 & 0.1 & 0.0 & 0.1 \\
\hline $20: 2 n-6$ & 0.3 & 0.3 & 0.1 & 0.1 \\
\hline $20: 3 n-6$ & 0.13 & 0.12 & 0.08 & 0.08 \\
\hline $20: 4 n-6$ & 1.2 & 1.0 & 0.9 & 0.7 \\
\hline$\sum n-6$ PUFA & 4.0 & 3.8 & $10 \cdot 4$ & 11.6 \\
\hline $18: 3 n-3$ & $1 \cdot 1$ & 1.2 & $15 \cdot 7$ & $19 \cdot 0$ \\
\hline $18: 4 n-3$ & 1.9 & $2 \cdot 1$ & 0.5 & 0.4 \\
\hline $20: 3 n-3$ & 0.14 & 0.15 & 0.06 & 0.04 \\
\hline $20: 4 n-3$ & 0.6 & 0.6 & 0.2 & 0.1 \\
\hline $20: 5 n-3$ & 7.8 & 8.0 & 3.5 & 2.5 \\
\hline $21: 5 n-3$ & 0.3 & 0.3 & 0.1 & 0.1 \\
\hline $22: 5 n-3$ & $1 \cdot 2$ & $1 \cdot 2$ & 0.7 & 0.5 \\
\hline $22: 6 n-3$ & $11 \cdot 7$ & $10 \cdot 8$ & $7 \cdot 2$ & 4.9 \\
\hline $\begin{array}{l}\sum_{\text {Ratios }} n-3 \text { LC-PUFA } \\
\end{array}$ & $24 \cdot 8$ & $24 \cdot 3$ & $28 \cdot 0$ & $27 \cdot 5$ \\
\hline SFA:PUFA & 1.0 & 0.9 & 0.8 & 0.7 \\
\hline$n-3: n-6$ & $6 \cdot 2$ & 6.4 & $2 \cdot 7$ & 2.4 \\
\hline Unsat. index & 181.4 & 179.5 & $169 \cdot 2$ & $160 \cdot 2$ \\
\hline
\end{tabular}

FO, fish oil; VO, blend of vegetable oils; $\mathrm{CH}$, carbohydrate; $\mathrm{CH}$ content, $0 \%(\mathrm{CH}-)$ or $20 \%(\mathrm{CH}+)$ gelatinised maize starch; $n-3$ LC-PUFA, $n-3$ long-chain PUFA; unsat. index, unsaturation index $=$ sum (fatty acid percentage $) \times$ (number of double bonds).

Lipid content in muscle was higher in the $\mathrm{CH}+$ group, whereas in the liver an increase in lipid content with carbohydrate intake was only evident when fish were fed VO-based diets (carbohydrate and lipid source interaction). Carbohydrate intake also increased the glycogen content in liver, but in muscle a similar effect was only recorded when fish were fed FO-based diets (carbohydrate and lipid source interaction). Dietary lipid source induced no changes on hepatic and muscular lipid content, and glycogen content in both tissues increased in the VO group when fish were fed $\mathrm{CH}$ - diets (carbohydrate and lipid source interaction). Hepatic cholesterol content was lower in $\mathrm{CH}+$ groups, but no differences were recorded in muscle cholesterol content. Dietary lipid source did not affect hepatic and muscular cholesterol content.

\section{Liver and muscle fatty acid profiles}

Muscle and liver FA composition were affected by diet composition and resembled the FA composition of the dietary lipid sources (Tables 5 and 6).

Except for muscle SFA content that was similar among $\mathrm{CH}+$ and $\mathrm{CH}-$ diets, liver and muscle FA profiles of fish fed the
$\mathrm{CH}+$ diets were characterised by a higher proportion of SFA (particularly 16:0) and MUFA (particularly 18:1n-9) and a lower proportion of $n-3$ PUFA and $n-6$ PUFA. In the liver, replacing $\mathrm{FO}$ by $\mathrm{VO}$ resulted in higher proportions of SFA (mainly 16:0) and $n$-6 PUFA (mainly 18:2n-6) and a lower proportion of $n-3$ PUFA (mainly $22: 6 n-3,20: 5 n-3,22: 5 n-3$ ) and MUFA (mainly $16: 1 n-7,20: 1 n-9,22: 1 n-9$ ).

The muscle of fish fed the VO diets presented a lower proportion of SFA (mainly 14:0) and MUFA (mainly 16:1n-7, $20: 1 n-9,22: 1 n-9)$ and an increased proportion of $n-6$ (mainly $18: 2 n-6$ ) and $n-3$ (mainly $18: 3 n-3$ ) PUFA.

\section{Plasma metabolites and enzyme activity}

After $18 \mathrm{~h}$ of feeding diets, $\mathrm{CH}+$ plasma glucose concentration was lower than in fish fed $\mathrm{CH}-$ diets (Table 7).

Plasma cholesterol was also lower in fish fed the $\mathrm{CH}+$ diet when combined with VO (carbohydrate and lipid source interaction). On the contrary, plasma TAG was higher in fish fed the $\mathrm{CH}+$ diet, but only in fish fed FO (carbohydrate and lipid source interaction). Plasma PL and cholesterol were lower in fish fed the VO diets. Among fish fed the $\mathrm{CH}+$ diets, plasma TAG was lower in the VO group than in the FO group (carbohydrate and lipid source interaction).

The $\mathrm{CH}+$ diet promoted an increase in FAS, G6PD and ME activities. G6PD activity was also responsive to dietary lipid source, being higher in VO diets (Table 7).

\section{Gene expression}

Hepatic transcript levels of glucokinase ( $G K)$, the first glycolytic enzyme, was higher in $\mathrm{CH}+$ than in $\mathrm{CH}$ - groups, whereas 6-phosphofructokinase ( $P F K$ ) transcript levels were not affected by dietary carbohydrate (Fig. 1). Hepatic transcript levels of phosphoenolpyruvate carboxykinase (PEPCK), the first key enzyme involved in gluconeogenesis, were lower in $\mathrm{CH}+$ than in $\mathrm{CH}$ - groups, whereas the opposite occurred for hepatic glucose 6-phosphatase (G6Pase) mRNA levels, the enzyme involved in the last step of gluconeogenesis. Among glycolytic and gluconeogenic enzymes, only hepatic G6Pase transcript levels were up-regulated by dietary VO. At the intestinal level, no transcriptional regulation by diet composition of $P F K$ and of PEPCK was observed (Fig. 1).

Hepatic and intestinal transcript levels of key enzymes involved in lipogenesis (FAS), $\beta$-oxidation (carnitine palmitoyltransferase 1A (CPT1A)) and PL synthesis (glycerol3-phosphate acyltransferase (GPAT)) were not affected by diet composition (Fig. 2).

With the exception of lanosterol $14 \alpha$-demethylase, cytochrome P450 51 (CYP51A1) transcript levels in the intestine, which were higher in $\mathrm{CH}+$ groups, no variation in hepatic or intestine transcript levels of proteins involved in cholesterol efflux (ATP-binding cassette G5 (ABCG5)), and cholesterol synthesis (7-dehydrocholesterol reductase (DHCR7); CYP51A1) and catabolism (liver X receptor $\alpha$ ( $L X R \alpha)$ ) were observed (Fig. 3).

The expression of genes encoding key proteins involved in the LC-PUFA-biosynthesis pathway ( $\Delta 6$ fatty acyl desaturaseliver isoform (FADS2); elongase 5 (elovl5)) were nutritionally 
Table 3. Growth performance and feed utilisation of gilthead sea bream fed the experimental diets (Mean values and standard deviations; $n$ 3)

\begin{tabular}{|c|c|c|c|c|c|c|c|c|c|c|c|}
\hline \multirow{4}{*}{$\begin{array}{l}\text { LS... } \\
\mathrm{CH}_{\ldots} . .\end{array}$} & \multicolumn{8}{|c|}{ Experimental diets } & & & \\
\hline & \multicolumn{4}{|c|}{$\mathrm{FO}$} & \multicolumn{4}{|c|}{ VO } & & & \\
\hline & \multicolumn{2}{|c|}{$\mathrm{CH}-$} & \multicolumn{2}{|c|}{$\mathrm{CH}+$} & \multicolumn{2}{|c|}{$\mathrm{CH}-$} & \multicolumn{2}{|c|}{$\mathrm{CH}+$} & \multicolumn{3}{|c|}{$P^{*}$} \\
\hline & Mean & SD & Mean & SD & Mean & SD & Mean & SD & $\mathrm{CH}$ & LS & $\mathrm{CH} \times \mathrm{LS}$ \\
\hline IBW (g) & $70 \cdot 8$ & 0.03 & 70.7 & 0.03 & $70 \cdot 8$ & 0.03 & 70.7 & 0.02 & 1.000 & 0.370 & 0.094 \\
\hline FBW (g) & $222 \cdot 3$ & 8.5 & $221 \cdot 8$ & $8 \cdot 8$ & 203.9 & $18 \cdot 8$ & $212 \cdot 1$ & 4.62 & 0.577 & 0.066 & 0.524 \\
\hline DGI† & $2 \cdot 37$ & 0.10 & $2 \cdot 37$ & $0 \cdot 10$ & $2 \cdot 16$ & 0.22 & 2.26 & 0.05 & 0.553 & 0.073 & 0.503 \\
\hline Feed intake(g/kg ABW $\neq$ per d) & 18.4 & 0.6 & $18 \cdot 4$ & 1.5 & $16 \cdot 7$ & 0.8 & $17 \cdot 3$ & 1.3 & 0.633 & 0.059 & 0.716 \\
\hline FE§ & 0.70 & 0.03 & 0.69 & 0.06 & 0.71 & 0.04 & 0.72 & 0.04 & 1.000 & 0.453 & 0.899 \\
\hline PER\| & 1.05 & 0.05 & $1 \cdot 38$ & 0.12 & 1.08 & 0.06 & 1.42 & 0.09 & $<0.001$ & 0.514 & 0.895 \\
\hline$N$ intake (g/kg ABW per d) & 1.95 & 0.08 & 1.49 & $0 \cdot 14$ & 1.77 & 0.18 & 1.39 & 0.13 & 0.001 & 0.122 & 0.637 \\
\hline$N$ retention (g/kg ABW per d)ף & 0.34 & 0.03 & 0.34 & 0.01 & 0.35 & 0.01 & 0.34 & 0.02 & 0.648 & 0.878 & 0.648 \\
\hline Lipid intake (g/kg ABW per d) & 3.37 & 0.14 & 3.39 & 0.31 & 3.04 & 0.30 & 3.15 & 0.29 & 0.681 & 0.104 & 0.757 \\
\hline Lipid retention ( $\mathrm{g} / \mathrm{kg}$ ABW per $\mathrm{d})^{\star \star}$ & $2 \cdot 21^{\mathrm{B}}$ & 0.05 & $2 \cdot 31$ & $0 \cdot 12$ & $1.84^{\mathrm{a}, \mathrm{A}}$ & 0.13 & $2 \cdot 36^{\mathrm{b}}$ & 0.07 & 0.001 & 0.023 & 0.006 \\
\hline
\end{tabular}

LS, lipid source; FO, fish oil; VO, blend of vegetable oils; $\mathrm{CH}$, carbohydrates; $\mathrm{CH}$ content, $0 \%(\mathrm{CH}-)$ or $20 \%(\mathrm{CH}+)$ gelatinised maize starch; IBW, initial body weight; FBW, final body weight; ABW, average body weight; FE, feed efficiency; PER, protein efficiency ratio.

a,b,A,B If interaction was significant, one-way ANOVA was performed for each factor, and means in the same line with different capital and small letters indicate significant differences $(P<0.05)$ between the two tested LP and two $\mathrm{CH}$ levels, respectively; means with no letters are not significantly different $(P>0.05)$

* Significant differences at $P<0.05$ two-way ANOVA.

† DGI: $\left(\left(\mathrm{FBW}^{1 / 3}-\mathrm{IBW}^{1 / 3}\right) /\right.$ time $\left.(\mathrm{d})\right) \times 100$

$\ddagger$ ABW: $($ IBW + FBW $) / 2$

$\S \mathrm{FE}$ : wet weight gain/dry feed intake.

II PER: wet weight gain/crude protein intake.

I $\mathrm{N}$ retentio $=((\mathrm{FBW} \times$ carcass $\mathrm{N}$ content $)-($ IBW $\times$ carcass $\mathrm{N}$ content $)) /(\mathrm{ABW} \times$ the number of days $)$

${ }^{\star *}$ Lipid retention $=(($ FBW $\times$ carcass lipid content $)-($ IBW $\times$ carcass lipid content $)) /(A B W \times$ the number of days $)$.

Table 4. Whole-body, liver and muscle composition (wet-weight basis), hepatosomatic (HSI) and viscerosomatic (VSI) indexes of gilthead sea bream fed the experimental diets*

(Mean values and standard deviations)

\begin{tabular}{|c|c|c|c|c|c|c|c|c|c|c|c|}
\hline \multirow{4}{*}{$\begin{array}{l}\text { LS... } \\
\text { CH... }\end{array}$} & \multicolumn{8}{|c|}{ Experimental diets } & & & \\
\hline & \multicolumn{4}{|c|}{$\mathrm{FO}$} & \multicolumn{4}{|c|}{ VO } & & & \\
\hline & \multicolumn{2}{|c|}{$\mathrm{CH}-$} & \multicolumn{2}{|c|}{$\mathrm{CH}+$} & \multicolumn{2}{|c|}{$\mathrm{CH}-$} & \multicolumn{2}{|c|}{$\mathrm{CH}+$} & \multicolumn{3}{|c|}{$P+$} \\
\hline & Mean & SD & Mean & SD & Mean & SD & Mean & SD & $\mathrm{CH}$ & LS & $\mathrm{CH} \times \mathrm{LS}$ \\
\hline \multicolumn{12}{|l|}{ Whole-body composition $\ddagger$} \\
\hline Protein (\%) & $16 \cdot 54$ & 0.73 & $16 \cdot 64$ & 0.18 & $17 \cdot 48$ & 0.46 & $16 \cdot 87$ & 0.82 & 0.484 & 0.134 & 0.341 \\
\hline Lipids (\%) & $14 \cdot 27^{\mathrm{B}}$ & 0.25 & 14.83 & 0.64 & $12 \cdot 75^{\mathrm{a}, \mathrm{A}}$ & 0.68 & $15 \cdot 35^{\mathrm{b}}$ & 0.38 & 0.001 & 0.123 & 0.009 \\
\hline $\mathrm{DM}(\%)$ & $35 \cdot 00$ & 0.23 & $36 \cdot 13$ & 0.62 & $34 \cdot 01$ & 0.72 & 35.94 & 0.28 & 0.001 & 0.078 & 0.208 \\
\hline Ash & 4.01 & 0.24 & 4.31 & 0.29 & 4.50 & 0.19 & 4.39 & 0.31 & 0.095 & 0.562 & 0.212 \\
\hline HSI§ & $1 \cdot 23$ & 0.17 & $1 \cdot 87$ & 0.20 & $1 \cdot 23$ & 0.22 & 1.96 & 0.31 & $<0.001$ & 0.478 & 0.343 \\
\hline VSIII & $6 \cdot 18$ & 0.68 & $6 \cdot 82$ & 0.89 & $6 \cdot 35$ & 0.94 & $6 \cdot 77$ & 1.05 & 0.003 & 0.755 & 0.528 \\
\hline \multicolumn{12}{|l|}{ Liver composition } \\
\hline Lipids (\%) & $13 \cdot 88$ & 3.25 & $12 \cdot 01$ & $2 \cdot 79$ & $10 \cdot 39^{a}$ & $3 \cdot 15$ & $13 \cdot 95^{\mathrm{b}}$ & 2.98 & 0.413 & 0.461 & 0.021 \\
\hline Cholesterol (\%) & 0.34 & 0.068 & 0.26 & 0.034 & 0.35 & 0.058 & 0.257 & 0.025 & $<0.001$ & 0.811 & 0.809 \\
\hline Glycogen (mg/g liver) & $73.49^{\mathrm{a}, \mathrm{A}}$ & $11 \cdot 75$ & $134.89^{b}$ & $7 \cdot 77$ & $89 \cdot 80^{\mathrm{a}, \mathrm{B}}$ & $11 \cdot 27$ & $125 \cdot 46^{\mathrm{b}}$ & $11 \cdot 21$ & $<0.001$ & 0.338 & 0.001 \\
\hline \multicolumn{12}{|l|}{ Muscle composition } \\
\hline Lipids (\%) & $8 \cdot 23$ & 1.06 & 9.85 & $2 \cdot 10$ & 8.08 & $1 \cdot 17$ & $9 \cdot 43$ & 1.05 & 0.020 & 0.661 & 0.877 \\
\hline Cholesterol (\%) & 0.097 & 0.008 & 0.088 & 0.008 & 0.09 & 0.009 & 0.097 & 0.010 & 0.811 & 0.831 & 0.051 \\
\hline Glycogen ( $\mu \mathrm{g} / \mathrm{g}$ muscle) & $12 \cdot 28^{\mathrm{a}, \mathrm{A}}$ & 2.47 & $29 \cdot 84^{\mathrm{b}}$ & $7 \cdot 89$ & $23 \cdot 66^{\mathrm{B}}$ & 3.40 & $31 \cdot 35$ & 8.57 & $<0.001$ & $<0.001$ & 0.001 \\
\hline
\end{tabular}

LS, lipid source; FO, fish oil; VO, blend of vegetable oils; $\mathrm{CH}$, carbohydrates; $\mathrm{CH}$ content, $0 \%(\mathrm{CH}-)$ or $20 \%(\mathrm{CH}+)$ gelatinised maize starch.

$\mathrm{a}, \mathrm{b}, \mathrm{A}, \mathrm{B}$ If interaction was significant, one-way ANOVA was performed for each factor, and means in the same line with different capital and small letters indicate significant differences $(P<0.05)$ between the two tested LS and two $\mathrm{CH}$ levels, respectively; means with no letters are not significantly different $(P>0.05)$.

* $n 3$ For whole-body composition; $n 6$ for lipids and cholesterol; $n 9$ for glycogen; $n 18$ for HSI and VSI.

$\dagger$ Significant differences at $P<0.05$ two-way ANOVA.

† Initial body composition on the fish: DM $28.96 \%$; protein $16.15 \%$; lipid $7.87 \%$; ash $5.84 \%$.

$\S \mathrm{HSI}$ : (liver weight/body weight) $\times 100$.

|| VSI: (viscera weight/body weight) $\times 100$.

regulated both in the liver and in the intestine (Fig. 4). In the liver, FADS2 transcript levels were upregulated by dietary carbohydrate and VO. Elovl5 expression in the liver was down-regulated in $\mathrm{CH}+$ groups, but in the intestine down-regulation was only evident in fish fed FO-based diets (carbohydrate and lipid source interaction). In addition, 
Table 5. Liver fatty acid profile (\% of total fatty acids) of gilthead sea bream fed the experimental diets* (Mean values and standard deviations; $n 6$ )

\begin{tabular}{|c|c|c|c|c|c|c|c|c|c|c|c|}
\hline \multirow{4}{*}{$\begin{array}{l}\text { LS... } \\
\mathrm{CH} . .\end{array}$} & \multicolumn{8}{|c|}{ Experimental diets } & & & \\
\hline & \multicolumn{4}{|c|}{ FO } & \multicolumn{4}{|c|}{ VO } & & & \\
\hline & \multicolumn{2}{|c|}{$\mathrm{CH}-$} & \multicolumn{2}{|c|}{$\mathrm{CH}+$} & \multicolumn{2}{|c|}{$\mathrm{CH}-$} & \multicolumn{2}{|c|}{$\mathrm{CH}+$} & \multicolumn{3}{|c|}{$P+$} \\
\hline & Mean & $\mathrm{SD}$ & Mean & SD & Mean & $\mathrm{SD}$ & Mean & SD & $\mathrm{CH}$ & LS & $\mathrm{CH} \times \mathrm{LS}$ \\
\hline $14: 0$ & $4 \cdot 3^{\mathrm{b}, \mathrm{B}}$ & 0.29 & $3.5^{\mathrm{a}, \mathrm{B}}$ & 0.46 & $2 \cdot 4^{\mathrm{A}}$ & 0.28 & $2 \cdot 8^{\mathrm{A}}$ & 0.32 & 0.185 & $<0.001$ & $<0.001$ \\
\hline $15: 0$ & 0.51 & 0.04 & 0.32 & 0.03 & 0.36 & 0.06 & 0.25 & 0.03 & $<0.001$ & $<0.001$ & 0.073 \\
\hline $16: 0$ & $19 \cdot 40$ & 0.87 & 22.29 & 0.69 & 22.03 & $2 \cdot 13$ & $25 \cdot 60$ & 1.48 & $<0.001$ & $<0.001$ & 0.643 \\
\hline $17: 0$ & 0.45 & 0.04 & 0.31 & 0.01 & 0.43 & 0.04 & 0.27 & 0.01 & $<0.001$ & 0.011 & 0.305 \\
\hline $18: 0$ & $4 \cdot 6^{\mathrm{a}, \mathrm{A}}$ & 0.38 & $6 \cdot 4^{\mathrm{b}}$ & 0.42 & $5 \cdot 6^{\mathrm{B}}$ & 0.50 & 5.99 & 0.30 & $<0.001$ & 0.074 & $<0.001$ \\
\hline $20: 0$ & 0.13 & 0.02 & 0.14 & 0.01 & 0.13 & 0.04 & 0.10 & 0.01 & 0.215 & 0.061 & 0.079 \\
\hline$\sum$ SFA & 29.48 & 0.87 & 33.03 & 0.76 & 31.00 & $2 \cdot 26$ & 35.02 & $1 \cdot 73$ & $<0.001$ & 0.011 & 0.737 \\
\hline $16: 1 n-7$ & $6 \cdot 5^{\mathrm{B}}$ & 0.32 & $6 \cdot 3^{\mathrm{B}}$ & 0.34 & $3 \cdot 5^{\mathrm{a}, \mathrm{A}}$ & 0.18 & $4 \cdot 4^{\mathrm{b}, \mathrm{A}}$ & $0 \cdot 21$ & 0.003 & $<0.001$ & $<0.001$ \\
\hline $18: 1 n-9$ & $22 \cdot 14$ & 1.62 & 27.92 & 1.24 & $27 \cdot 07$ & 1.87 & $32 \cdot 20$ & 1.51 & $<0.001$ & $<0.001$ & 0.475 \\
\hline $20: 1 n-9$ & 3.64 & 0.59 & 3.53 & 0.31 & 0.77 & 0.20 & 0.48 & 0.03 & 0.034 & $<0.001$ & 0.108 \\
\hline $22: 1 n-9$ & $2 \cdot 16$ & 0.35 & 2.01 & 0.36 & 0.30 & 0.17 & 0.08 & 0.03 & 0.008 & $<0.001$ & 0.068 \\
\hline$\sum$ MUFA & 34.68 & 1.73 & 39.91 & 1.34 & 31.73 & $2 \cdot 17$ & $37 \cdot 24$ & 1.61 & $<0.001$ & 0.001 & 0.795 \\
\hline $18: 2 n-6$ & $2 \cdot 41$ & $0 \cdot 16$ & 1.97 & 0.22 & $8 \cdot 21$ & 0.45 & 7.90 & 0.34 & 0.002 & $<0.001$ & 0.109 \\
\hline $18: 3 n-6$ & $0 \cdot 18^{\mathrm{B}}$ & 0.06 & $0.19^{\mathrm{B}}$ & 0.01 & $0 \cdot 10^{\mathrm{a}, \mathrm{A}}$ & 0.05 & $0.30^{\mathrm{b}, \mathrm{A}}$ & 0.05 & $<0.001$ & 0.864 & $<0.001$ \\
\hline $20: 2 n-6$ & $0.26^{b}$ & 0.02 & $0.21^{\mathrm{a}, \mathrm{B}}$ & 0.01 & $0.25^{b}$ & 0.05 & $0.15^{\mathrm{a}, \mathrm{A}}$ & 0.01 & $<0.001$ & 0.003 & 0.029 \\
\hline $20: 3 n-6$ & 0.14 & 0.02 & 0.11 & 0.01 & 0.08 & 0.04 & 0.11 & 0.02 & 0.515 & 0.031 & 0.071 \\
\hline $20: 4 n-6$ & $1.4^{\mathrm{b}}$ & 0.22 & $1 \cdot 0^{\mathrm{a}, \mathrm{B}}$ & 0.11 & $1.5^{\mathrm{b}}$ & 0.35 & $0.64^{\mathrm{a}, \mathrm{A}}$ & 0.06 & $<0.001$ & 0.003 & 0.003 \\
\hline$\sum n-6$ PUFA & $4 \cdot 61$ & 0.31 & 3.56 & 0.21 & $10 \cdot 08$ & $0 \cdot 70$ & $9 \cdot 10$ & 0.30 & $<0.001$ & $<0.001$ & 0.141 \\
\hline $18: 3 n-3$ & $0.91^{b, A}$ & 0.09 & $0.69^{\mathrm{a}, \mathrm{A}}$ & 0.08 & $11 \cdot 1^{\mathrm{B}}$ & 0.82 & $10 \cdot 5^{\mathrm{B}}$ & 0.84 & $<0.001$ & $<0.001$ & 0.012 \\
\hline $18: 4 n-3$ & $1 \cdot 0^{\mathrm{b}, \mathrm{B}}$ & 0.12 & $0.84^{\mathrm{a}, \mathrm{B}}$ & 0.10 & $0.30^{\mathrm{a}, \mathrm{A}}$ & 0.04 & $0.69^{b, A}$ & 0.12 & 0.002 & $<0.001$ & $<0.001$ \\
\hline $20: 3 n-3$ & $0 \cdot 18^{\mathrm{b}, \mathrm{A}}$ & 0.03 & $0.13^{\mathrm{a}, \mathrm{A}}$ & 0.02 & $0.65^{\mathrm{b}, \mathrm{B}}$ & 0.16 & $0.37^{\mathrm{a}, \mathrm{B}}$ & 0.04 & $<0.001$ & $<0.001$ & 0.020 \\
\hline $20: 4 n-3$ & 0.85 & 0.11 & 0.76 & 0.06 & 0.36 & 0.06 & 0.34 & 0.06 & 0.131 & $<0.001$ & 0.412 \\
\hline $20: 5 n-3$ & $5 \cdot 3^{\mathrm{b}, \mathrm{B}}$ & 0.36 & $4 \cdot 2^{\mathrm{a}, \mathrm{B}}$ & 0.30 & $2 \cdot 5^{\mathrm{b}, \mathrm{A}}$ & 0.42 & $1 \cdot 2^{\mathrm{a}, \mathrm{A}}$ & 0.05 & $<0.001$ & $<0.001$ & 0.003 \\
\hline $21: 5 n-3$ & 0.27 & 0.03 & 0.21 & 0.05 & 0.04 & 0.05 & 0.00 & 0.01 & 0.023 & $<0.001$ & 0.439 \\
\hline $22: 5 n-3$ & $2 \cdot 6^{\mathrm{b}, \mathrm{B}}$ & 0.31 & $2 \cdot 0^{\mathrm{a}, \mathrm{B}}$ & 0.24 & $1 \cdot 3^{\mathrm{b}, \mathrm{A}}$ & 0.36 & $0.47^{\mathrm{a}, \mathrm{A}}$ & 0.05 & $<0.001$ & $<0.001$ & 0.017 \\
\hline $22: 6 n-3$ & $14 \cdot 7^{\mathrm{b}, \mathrm{B}}$ & 1.44 & $9 \cdot 6^{\mathrm{a}, \mathrm{B}}$ & 0.68 & $8 \cdot 6^{\mathrm{b}, \mathrm{A}}$ & 1.55 & $3 \cdot 2^{\mathrm{a}, \mathrm{A}}$ & 0.11 & $<0.001$ & $<0.001$ & 0.016 \\
\hline$\sum n$-3PUFA & $25 \cdot 82$ & 1.63 & 18.44 & 1.02 & $24 \cdot 89$ & $1 \cdot 80$ & $16 \cdot 74$ & $0 \cdot 87$ & $<0.001$ & 0.024 & 0.398 \\
\hline \multicolumn{12}{|l|}{ Ratios } \\
\hline SFA:PUFA & 0.93 & 0.07 & 1.42 & 0.08 & 0.87 & 0.10 & $1 \cdot 33$ & 0.11 & $<0.001$ & 0.060 & 0.846 \\
\hline$n-3: n-6$ & $5 \cdot 6^{\mathrm{b}, \mathrm{B}}$ & $0 \cdot 18$ & $5 \cdot 2^{\mathrm{a}, \mathrm{B}}$ & 0.28 & $2 \cdot 5^{\mathrm{b}, \mathrm{A}}$ & 0.20 & $1 \cdot 8^{\mathrm{a}, \mathrm{A}}$ & 0.05 & $<0.001$ & $<0.001$ & 0.007 \\
\hline Unsat. index & 191.02 & 8.95 & 151.51 & 5.08 & $159 \cdot 30$ & $16 \cdot 39$ & $122 \cdot 88$ & 3.41 & $<0.001$ & $<0.001$ & 0.705 \\
\hline
\end{tabular}

LS, lipid source; FO, fish oil; VO, blend of vegetable oils; $\mathrm{CH}$, carbohydrates; $\mathrm{CH}$ content, $0 \%(\mathrm{CH}-)$ or $20 \%(\mathrm{CH}+)$ gelatinised maize starch; $n$-3 LC-PUFA, $n$-3 long-chain PUFA; unsat. index, unsaturation index $=$ sum (fatty acid percentage) $\times$ (number of double bonds).

$\mathrm{a}, \mathrm{b}, \mathrm{A}, \mathrm{B}$ If interaction was significant, one-way ANOVA was performed for each factor, and means in the same line with different capital and small letters indicate significant differences $(P<0.05)$ between the two tested LS and two $\mathrm{CH}$ levels, respectively; means with no letters are not significantly different $(P>0.05)$.

${ }^{*} \mathrm{FA} \geq 0.02 \%,<0.02 \%$ was not considered in the table.

$\dagger$ Significant differences at $P<0.05$ two-way ANOVA.

intestinal elovl 5 mRNA levels increased in VO groups when fed a carbohydrate-rich diet (carbohydrate and lipid source interaction).

In the liver transcription of PPAR $\alpha$ and PPAR $\gamma$ was downregulated in $\mathrm{CH}+$ groups while in the intestine down-regulation of PPAR $\beta$ transcript levels in $\mathrm{CH}+$ groups was only observed when fish were fed the VO diet (carbohydrate and lipid source interaction) (Fig. 4). Additionally, intestine from the $\mathrm{CH}-$ groups exhibited an up-regulation of $P P A R \beta$ transcripts when fish were fed the VO diet (carbohydrate and lipid source interaction).

\section{Discussion}

\section{Effects of dietary carbohydrates}

Although gilthead sea bream is a carnivorous species (trophic level $3 \cdot 3-3.5$ according to Fish Base), it tolerates up to $20 \%$ dietary starch without detrimental effects in growth performance and feed efficiency. These results support previous evidences ${ }^{(61,62)}$ and indicate the possibility to reduce aquafeed costs and alleviate the overexploitation of fisheries marine resources through the use of the carbohydrate component in gilthead sea bream diets.

The low glycaemia values at $18 \mathrm{~h}$ after feeding are in accordance with results of Peres et al. ${ }^{(63)}$ that observed in a glucose tolerance test that seabream was able to restore glucose levels within $12 \mathrm{~h}$ after receiving an overdose of glucose.

Hepatic GK is a key player in blood glucose homoeostasis by catalysing the phosphorylation of glucose and providing the first substrate for glycolysis, glycogenesis and the pentose phosphate pathway ${ }^{(64)}$. An induction of hepatic $G K$ transcripts by dietary carbohydrates was recorded in the present study, as in a previous study in this species ${ }^{(65)}$. On the contrary, dietary carbohydrates did not affect transcription levels of hepatic PFK, another key glycolytic enzyme. 
Table 6. Muscle fatty acid profile (expressed as \% of total fatty acids) of gilthead sea bream fed the experimental diets* (Mean values and standard deviations; $n 6$ )

\begin{tabular}{|c|c|c|c|c|c|c|c|c|c|c|c|}
\hline \multirow{4}{*}{$\begin{array}{l}\text { LS... } \\
\mathrm{CH} \ldots\end{array}$} & \multicolumn{8}{|c|}{ Experimental diets } & & & \\
\hline & \multicolumn{4}{|c|}{ FO } & \multicolumn{4}{|c|}{ VO } & & & \\
\hline & \multicolumn{2}{|c|}{$\mathrm{CH}-$} & \multicolumn{2}{|c|}{$\mathrm{CH}+$} & \multicolumn{2}{|c|}{$\mathrm{CH}-$} & \multicolumn{2}{|c|}{$\mathrm{CH}+$} & \multicolumn{3}{|c|}{$P \dagger$} \\
\hline & Mean & SD & Mean & SD & Mean & SD & Mean & SD & $\mathrm{CH}$ & LS & $\mathrm{CH} \times \mathrm{LS}$ \\
\hline $14: 0$ & 4.76 & 0.17 & 4.59 & 0.19 & $2 \cdot 72$ & 0.17 & $2 \cdot 39$ & 0.14 & 0.001 & $<0.001$ & 0.108 \\
\hline $15: 0$ & 0.52 & 0.02 & 0.43 & 0.01 & 0.34 & 0.01 & 0.24 & 0.01 & $<0.001$ & $<0.001$ & 0.002 \\
\hline $16: 0$ & $19 \cdot 24$ & 0.54 & 19.99 & 0.71 & $19 \cdot 06$ & 0.82 & 19.63 & 0.33 & 0.018 & 0.307 & 0.754 \\
\hline $17: 0$ & 0.39 & 0.02 & 0.30 & 0.01 & 0.32 & 0.02 & 0.23 & 0.01 & $<0.001$ & $<0.001$ & 0.179 \\
\hline $18: 0$ & 3.30 & 0.22 & 3.72 & $0 \cdot 17$ & 3.59 & 0.15 & 4.01 & 0.10 & $<0.001$ & $<0.001$ & 0.875 \\
\hline $20: 0$ & 0.15 & 0.01 & 0.14 & 0.00 & 0.20 & 0.01 & 0.18 & 0.01 & 0.013 & $<0.001$ & 0.238 \\
\hline$\sum$ SFA & $28 \cdot 41$ & 0.58 & $29 \cdot 22$ & 0.80 & $26 \cdot 32$ & 1.04 & $26 \cdot 77$ & 0.56 & 0.058 & $<0.001$ & 0.585 \\
\hline $16: 1 n-7$ & 7.92 & 0.16 & 7.80 & 0.12 & 4.69 & 0.44 & $4 \cdot 18$ & 0.20 & 0.009 & $<0.001$ & 0.058 \\
\hline $18: 1 n-9$ & $24.42^{\mathrm{a}, \mathrm{A}}$ & 0.85 & $25 \cdot 76^{\mathrm{b}, \mathrm{A}}$ & 0.32 & $28 \cdot 76^{\mathrm{a}, \mathrm{B}}$ & 0.39 & $32 \cdot 23^{\mathrm{b}, \mathrm{B}}$ & 0.35 & $<0.001$ & $<0.001$ & $<0.001$ \\
\hline $20: 1 n-9$ & $3.83^{\mathrm{B}}$ & 0.29 & $4.06^{\mathrm{B}}$ & 0.11 & $1 \cdot 14^{\mathrm{b}, \mathrm{A}}$ & 0.03 & $0.95^{\mathrm{a}, \mathrm{A}}$ & 0.08 & 0.020 & $<0.001$ & $<0.001$ \\
\hline $22: 1 n-9$ & $2 \cdot 13^{\mathrm{a}, \mathrm{B}}$ & 0.18 & $2 \cdot 40^{\mathrm{b}, \mathrm{B}}$ & 0.12 & $0.55^{\mathrm{b}, \mathrm{A}}$ & 0.04 & $0.41^{\mathrm{a}, \mathrm{A}}$ & 0.04 & 0.778 & $<0.001$ & $<0.001$ \\
\hline$\sum$ MUFA & $38.50^{\mathrm{a}, \mathrm{B}}$ & 0.48 & $40 \cdot 20^{\mathrm{b}, \mathrm{B}}$ & 0.28 & $35 \cdot 28^{\mathrm{a}, \mathrm{A}}$ & 0.79 & $37.93^{\mathrm{b}, \mathrm{A}}$ & 0.30 & $<0.001$ & $<0.001$ & 0.030 \\
\hline $18: 2 n-6$ & $4 \cdot 19$ & 0.32 & 3.66 & 0.47 & $9 \cdot 13$ & 0.55 & $9 \cdot 25$ & 0.35 & 0.140 & $<0.001$ & 0.051 \\
\hline $18: 3 n-6$ & 0.28 & 0.04 & 0.26 & 0.06 & 0.20 & 0.07 & 0.25 & 0.04 & 0.429 & 0.059 & 0.081 \\
\hline $20: 2 n-6$ & 0.26 & 0.01 & 0.23 & 0.01 & 0.25 & 0.01 & 0.21 & 0.01 & $<0.001$ & 0.004 & 0.241 \\
\hline $20: 3 n-6$ & $0.14^{\mathrm{b}, \mathrm{B}}$ & 0.02 & $0.11^{\mathrm{a}, \mathrm{A}}$ & 0.01 & $0.11^{\mathrm{A}}$ & 0.01 & $0.12^{\mathrm{B}}$ & 0.02 & 0.026 & 0.180 & 0.007 \\
\hline $20: 4 n-6$ & $0.98^{\mathrm{b}, \mathrm{B}}$ & 0.03 & $0.79^{a, B}$ & 0.04 & $0.87^{\mathrm{b}, \mathrm{A}}$ & 0.07 & $0.59^{\mathrm{a}, \mathrm{A}}$ & 0.04 & $<0.001$ & $<0.001$ & 0.007 \\
\hline$\sum n-6$ PUFA & $5 \cdot 85^{\mathrm{b}, \mathrm{A}}$ & 0.30 & $5.04^{\mathrm{a}, \mathrm{A}}$ & 0.52 & $10.56^{\mathrm{B}}$ & 0.59 & $10 \cdot 41^{\mathrm{B}}$ & 0.42 & 0.013 & $<0.001$ & 0.047 \\
\hline $18: 3 n-3$ & $1 \cdot 10^{\mathrm{b}, \mathrm{A}}$ & 0.03 & $1.03^{\mathrm{a}, \mathrm{A}}$ & 0.06 & $11 \cdot 19^{\mathrm{a}, \mathrm{B}}$ & 0.94 & $12 \cdot 60^{\mathrm{b}, \mathrm{B}}$ & 0.58 & 0.301 & $<0.001$ & 0.001 \\
\hline $18: 4 n-3$ & 1.21 & 0.09 & 1.27 & 0.08 & 0.45 & 0.03 & 0.45 & 0.04 & 0.410 & $<0.001$ & 0.206 \\
\hline $20: 3 n-3$ & 0.15 & 0.02 & 0.14 & 0.02 & 0.42 & 0.04 & 0.40 & 0.03 & 0.071 & $<0.001$ & 0.884 \\
\hline $20: 4 n-3$ & 0.75 & 0.03 & 0.69 & 0.03 & 0.38 & 0.02 & 0.35 & 0.03 & 0.001 & $<0.001$ & 0.723 \\
\hline $20: 5 n-3$ & $5 \cdot 10^{\mathrm{B}}$ & 0.13 & $5 \cdot 01^{B}$ & 0.07 & $2 \cdot 75^{\mathrm{b}, \mathrm{A}}$ & 0.16 & $1.87^{\mathrm{a}, \mathrm{A}}$ & 0.11 & $<0.001$ & $<0.001$ & $<0.001$ \\
\hline $21: 5 n-3$ & $0.25^{\mathrm{B}}$ & 0.01 & $0.23^{\mathrm{B}}$ & 0.02 & $0.11^{\mathrm{b}, \mathrm{A}}$ & 0.02 & $0.03^{\mathrm{a}, \mathrm{A}}$ & 0.04 & 0.002 & $<0.001$ & 0.006 \\
\hline $22: 5 n-3$ & $2 \cdot 15^{\mathrm{b}, \mathrm{B}}$ & 0.17 & $1.86^{a, B}$ & 0.12 & $1.44^{\mathrm{b}, \mathrm{A}}$ & 0.08 & $0.99^{\mathrm{a}, \mathrm{A}}$ & 0.04 & $<0.001$ & $<0.001$ & 0.007 \\
\hline $22: 6 n-3$ & $11 \cdot 32^{\mathrm{b}, \mathrm{B}}$ & 0.86 & $10 \cdot 17^{\mathrm{a}, \mathrm{B}}$ & 0.09 & $8.37^{\mathrm{b}, \mathrm{A}}$ & 0.54 & $5 \cdot 82^{\mathrm{a}, \mathrm{A}}$ & 0.38 & $<0.001$ & $<0.001$ & 0.001 \\
\hline$\sum n-3$ LC PUFA & $22 \cdot 02$ & 1.05 & $20 \cdot 39$ & 0.24 & $25 \cdot 12$ & $1 \cdot 26$ & $22 \cdot 50$ & 0.52 & $<0.001$ & $<0.001$ & 0.212 \\
\hline \multicolumn{12}{|l|}{ Ratios } \\
\hline SFA:PUFA & 0.97 & 0.05 & & 0.05 & & 0.06 & 0.80 & 0.03 & $<0.001$ & $<0.001$ & 0.423 \\
\hline$n-3: n-6$ & $3 \cdot 78^{\mathrm{B}}$ & 0.29 & $4.08^{\mathrm{B}}$ & 0.46 & $2 \cdot 38^{\mathrm{b}, \mathrm{A}}$ & 0.15 & $2 \cdot 16^{\mathrm{a}, \mathrm{A}}$ & 0.11 & 0.943 & $<0.001$ & 0.023 \\
\hline Unsat. index & $173 \cdot 26^{b}$ & 5.55 & $163.51^{\mathrm{a}, \mathrm{B}}$ & 1.57 & $170 \cdot 48^{\mathrm{b}}$ & $5 \cdot 27$ & $153.53^{\mathrm{a}, \mathrm{A}}$ & 2.59 & $<0.001$ & 0.001 & 0.029 \\
\hline
\end{tabular}

LS, lipid source; FO, fish oil; VO, blend of vegetable oils; $\mathrm{CH}$, carbohydrates; $\mathrm{CH}$ content, $0 \%(\mathrm{CH}-)$ or $20 \%(\mathrm{CH}+)$ gelatinised maize starch; $n-3 \mathrm{LC}$-PUFA, $n-3$ long-chain PUFA; unsat. index, unsaturation index $=$ sum (fatty acid percentage) $\times$ (number of double bonds).

a,b,A,B If interaction was significant, one-way ANOVA was performed for each factor, and means in the same line with different capital and small letters indicate significant differences $(P<0.05)$ between the two tested LS and two $\mathrm{CH}$ levels, respectively; means with no letters are not significantly different $(P>0.05)$.

${ }^{*} \mathrm{FA}>0.02 \%,<0.02 \%$ was not considered in the table.

$\dagger$ Significant differences at $P<0.05$ two-way ANOVA.

Hepatic transcript levels of $P E P C K$, the first key enzyme of gluconeogenesis, were down-regulated by dietary carbohydrate, but the transcript levels of G6Pase, another key enzyme of gluconeogenesis, was up-regulated by dietary carbohydrates. Although a similar response of hepatic G6Pase by dietary carbohydrate at the transcriptional level was previously reported in carnivorous rainbow trout ${ }^{(24)}$, present data apparently contradict results of Panserat et al. ${ }^{(66)}$ in this species, who observed that G6Pase and FBPase were down-regulated by dietary carbohydrates at the transcriptional level, whereas PEPCK was not affected. At the enzymic activity level, carbohydrates in gilthead sea bream diets induced minor effects ${ }^{(62,67)}$ or even an increase $^{(61)}$ in the hepatic activities of gluconeogenesis enzymes. Recent studies pointed out that the intestine has important functions in glucose homoeostasis ${ }^{(68,69)}$. However, present results do not support that assumption, as expression of glycolytic $(P F K)$ and gluconeogenesis (PEPCK) enzymes in this tissue did not respond to dietary carbohydrate.
In parallel with the hepatic up-regulation of $G K$ transcripts, increased HSI and VSI were recorded in fish fed carbohydraterich diets, which may indicate that the hepatic glucose pool was directed towards glycogen and/or FA synthesis. Indeed, in liver, an increased deposition of glycogen and a higher lipogenic potential, indicated by FAS, G6PD and ME activities, were recorded in $\mathrm{CH}+$ groups. Increased HSI may have unwanted physiological effects; therefore, histomorphological analysis of liver should be considered in future studies to discard any histopathological damage induced by diet.

Despite the fact that lipogenesis was nutritionally regulated by dietary carbohydrates, the contribution to the overall lipid deposition in liver or whole body of fish fed FO-based diet seemed to be minor, as lipids in liver and whole body increased with carbohydrate intake only under a VO-based diet regimen.

Recently, it was suggested that dietary carbohydrates have a role in cholesterol biosynthesis by inducing (at least at a molecular level) the capacity to produce $\mathrm{it}^{(7)}$. In the present 
Table 7. Plasma metabolite concentrations $(n 18)(\mathrm{mmol} / \mathrm{l})$ and enzymatic activity ( $\mu / \mathrm{mg}$ protein) of selected enzymes involved in lipogenesis $(n 9)$ in gilthead sea bream fed the experimental diets

(Mean values and standard deviations)

\begin{tabular}{|c|c|c|c|c|c|c|c|c|c|c|c|}
\hline \multirow{4}{*}{$\begin{array}{l}\text { LS... } \\
\mathrm{CH} . . .\end{array}$} & \multicolumn{8}{|c|}{ Experimental diets } & & & \\
\hline & \multicolumn{4}{|c|}{$\mathrm{FO}$} & \multicolumn{4}{|c|}{ VO } & & & \\
\hline & \multicolumn{2}{|c|}{$\mathrm{CH}-$} & \multicolumn{2}{|c|}{$\mathrm{CH}+$} & \multicolumn{2}{|c|}{$\mathrm{CH}-$} & \multicolumn{2}{|c|}{$\mathrm{CH}+$} & \multicolumn{3}{|c|}{$P^{\star}$} \\
\hline & Mean & SD & Mean & SD & Mean & SD & Mean & SD & $\mathrm{CH}$ & LS & $\mathrm{CH} \times \mathrm{LS}$ \\
\hline \multicolumn{12}{|c|}{ Plasma metabolites } \\
\hline GLU & 3.73 & 0.60 & 3.16 & 0.49 & 3.47 & 0.34 & $3 \cdot 19$ & 0.46 & $<0.001$ & 0.312 & 0.203 \\
\hline $\mathrm{CHOL}$ & $7 \cdot 37^{B}$ & 0.53 & $7 \cdot 25^{\mathrm{B}}$ & 0.95 & $6 \cdot 80^{\mathrm{b}, \mathrm{A}}$ & 0.99 & $5.82^{\mathrm{a}, \mathrm{A}}$ & 0.65 & 0.005 & $<0.001$ & 0.027 \\
\hline TAG & $2 \cdot 87^{\mathrm{a}}$ & 0.63 & $3.73^{\mathrm{b}, \mathrm{B}}$ & 0.73 & $3 \cdot 15$ & 0.86 & $3 \cdot 15^{\mathrm{A}}$ & 0.67 & 0.015 & 0.383 & 0.016 \\
\hline PL & $15 \cdot 37$ & 1.47 & $15 \cdot 45$ & 1.44 & 14.46 & 2.54 & 13.42 & 1.26 & 0.246 & 0.001 & 0.178 \\
\hline \multicolumn{12}{|c|}{ Enzyme activity } \\
\hline G6PD & $140 \cdot 1$ & $36 \cdot 3$ & $203 \cdot 7$ & 49.1 & $198 \cdot 6$ & $27 \cdot 6$ & $240 \cdot 0$ & 68.6 & 0.004 & 0.009 & 0.511 \\
\hline ME & 6.97 & $3 \cdot 10$ & 9.58 & 3.49 & 5.51 & 1.96 & 11.63 & 4.37 & 0.001 & 0.791 & 0.130 \\
\hline FAS & $4 \cdot 28$ & $2 \cdot 76$ & $7 \cdot 38$ & 1.75 & 5.46 & $2 \cdot 91$ & 8.60 & 3.61 & 0.003 & 0.225 & 0.981 \\
\hline
\end{tabular}

LS, lipid source; FO, fish oil; VO, blend of vegetable oils; $\mathrm{CH}$, carbohydrates; $\mathrm{CH}$ content, $0 \%(\mathrm{CH}-)$ or $20 \%(\mathrm{CH}+)$ gelatinised maize starch; $\mathrm{GLU}$, glucose; $\mathrm{CHOL}$, total cholesterol; PL, phospholipids; G6PD, glucose-6-phosphate dehydrogenase; ME, malic enzyme; FAS, fatty acid synthase.

a,b,A,B If interaction was significant, one-way ANOVA was performed for each factor, and means in the same line with different capital and small letters indicate significant differences $(P<0.05)$ between the two tested LS and two $\mathrm{CH}$ levels, respectively; means with no letters are not significantly different $(P>0.05)$.

* Significant differences at $P<0.05$ two-way ANOVA.

study, it was observed that gilthead sea bream fed carbohydraterich diets exhibited reduced liver and plasma (only in VOCH+ group) cholesterol content. Furthermore, an increased expression at the intestinal level of one gene encoding for an enzyme involved in the last steps of cholesterol biosynthesis, CYP51A1, was also observed. We hypothesise that the depletion of the cholesterol pool was promoted by a lower dietary input of cholesterol, and the up-regulation of CYP51A1 may reflect an increased synthesis of sterols by the intestinal cells in response to the low dietary supply. Metabolic adjustments in de novo cholesterol biosynthesis in response to the dietary load of cholesterol were previously described in Atlantic salmon ${ }^{(70)}$.

In this work, we demonstrated a transcriptional up-regulation by dietary carbohydrate of liver FADS2, a key desaturase involved in LC-PUFA synthesis. With the up-regulation of FADS2 gene, it was expected to observe increased transcript levels of elovl5, a fatty acyl elongase involved in LC-PUFA biosynthesis. However, and somehow surprisingly, the reverse pattern was observed both in the intestine (only in $\mathrm{FOCH}+$ group) and in the liver of gilthead sea bream fed $\mathrm{CH}+$ diets. In the present state of knowledge, we have no clear explanation for these conflicting results, and therefore further studies are needed to better understand the apparent atypical molecular regulation of some enzymes of the LC-PUFA synthesis pathway by dietary carbohydrate in gilthead sea bream. Irrespective of the regulation of FADS 2 expression, increased liver or muscle $n$-3 LC-PUFA content of gilthead sea bream fed $\mathrm{CH}+$ diets were not observed. On the contrary, reduced $n$ - 3 LC-PUFA content in $\mathrm{CH}+$ groups was recorded, which could be related to an increase in SFA derived by lipogenesis from carbohydrates, as previously reported in other species such as rainbow trout and European sea bass ${ }^{(7,71)}$.

PPAR are a family of nuclear receptors that have three isoforms in mammals known as $\operatorname{PPAR} \alpha, \operatorname{PPAR} \beta$ and PPAR $\gamma$ that play key roles in regulation of lipid metabolism ${ }^{(72)}$. It is believed that PPAR control metabolic pathways that support FA $\beta$-oxidation (especially $\operatorname{PPAR} \alpha$ ), tissue lipid deposition and lipogenesis (especially PPAR $\gamma$ ), and the overall lipid homoeostasis (especially PPAR $\beta$ ), mainly by means of the action of its ligands (such as FA and their derivatives, and also glucose $)^{(72,73)}$. In this study, despite the down-regulation of $P P A R \alpha, \beta$ gene expression in the liver and of PPAR $\beta$ in the intestine (only in VO diets) by dietary carbohydrates, gene expression of CPT1, a marker of mitochondrial FA $\beta$-oxidation, was unaffected both in the liver and intestine of fish fed $\mathrm{CH}+$ diets. In previous studies, a synchronised regulation between piscine $P P A R \alpha, \beta$ gene expression and the activity or expression of other enzymes involved in FA $\beta$-oxidation, such as acyl-CoA oxidase and L-3-hydroxyacyl-CoA dehydrogenase, was described $^{(74,75)}$. However, present results are not completely unexpected, as in a number of studies the expression of the CPT1 gene was not nutritionally regulated or directly linked to the FA $\beta$-oxidation capacity ${ }^{(24,37,76)}$. Furthermore, FA $\beta$-oxidation and lipogenesis are two pathways usually regulated in opposite directions $^{(77,78)}$. Therefore, as lipogenic potential increased in liver of fish fed $\mathrm{CH}+$ diets, it is possible that $\mathrm{FA} \beta$-oxidation might have been repressed. Additional data on the activity or expression of the other FA $\beta$-oxidation related enzymes would be necessary to confirm this hypothesis.

In the above discussion, differences in metabolic responses were assumed to be related to differences in dietary carbohydrate ( $0 v$ v 17-18\%). However, a potential effect of dietary protein cannot be discarded, particularly in relation to regulation of gluconeogenesis and lipogenesis, as there is increasing evidence that protein and amino acids also modulate these pathways ${ }^{(79-82)}$.

\section{Effects of dietary lipid source}

In the present study, gilthead sea bream performed as well as in previous studies with gilthead sea bream juveniles in which 
(a)

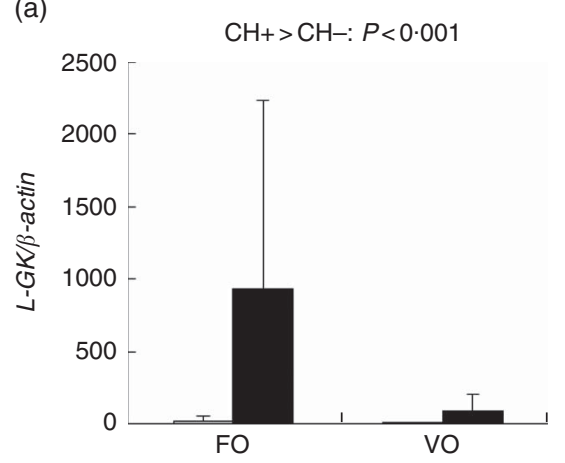

$\mathrm{CH}+<\mathrm{CH}-: \mathrm{P}=0.014$

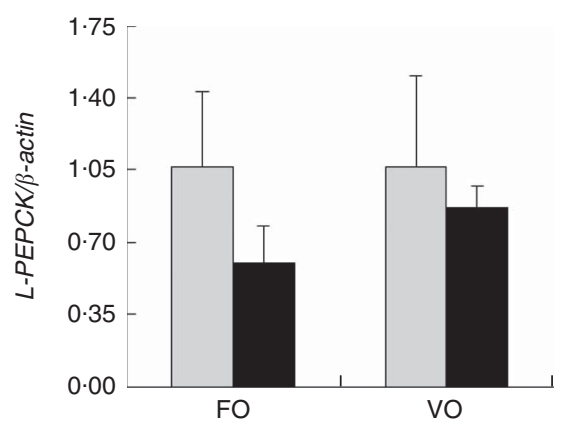

(b)

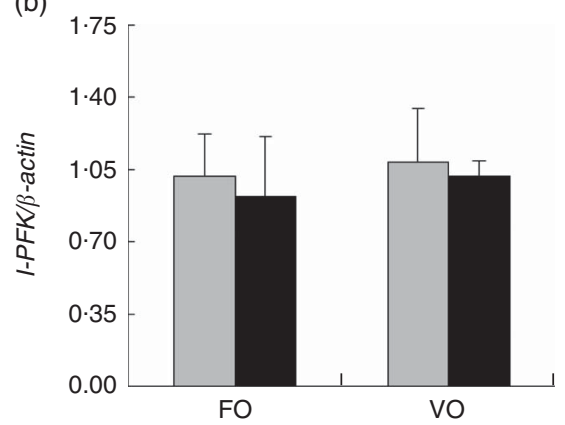

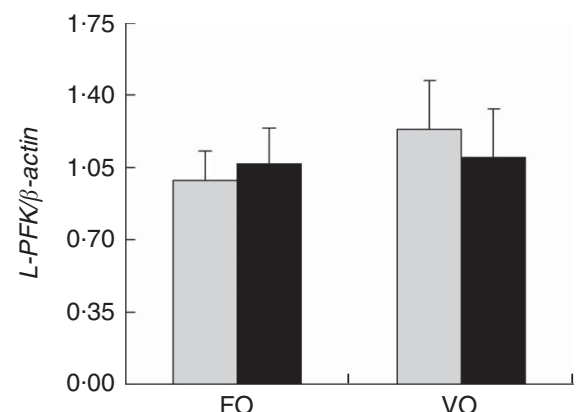

$\mathrm{CH}+>\mathrm{CH}-: \mathrm{P}=0.036$ $\mathrm{VO}>\mathrm{FO}: P=0.009$
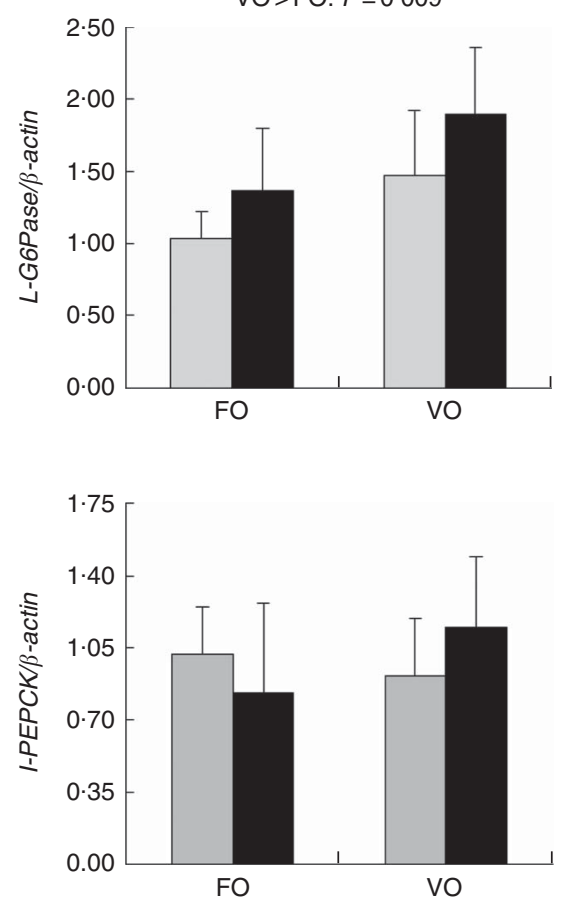

Fig. 1. mRNA levels of genes involved in glycolysis (glucokinase (GK), 6-phosphofructokinase (PFK)) and gluconeogenesis (phosphoenolpyruvate carboxykinase (PEPCK), glucose 6-phosphatase (G6Pase)) in the (a) liver and (b) intestine of gilthead sea bream fed the experimental diets. Expression values are normalised by $\beta$-actin-expressed transcripts. Fish oil (FO), blend of vegetable oils (VO); carbohydrate content: $0 \%(\square, \mathrm{CH}-)$ or $20 \%(\square, \mathrm{CH}+)$ gelatinised maize starch. Relative fold difference between treatments are presented as means $(n 6)$, with their standard deviations. Significant differences at $P<0.05$ (two-way ANOVA).

dietary FO was partially $(70 \%)$ replaced by $\mathrm{VO}$ in $\mathrm{FM}^{(83,84)}$ or plant protein ${ }^{(85,86)}$-based diets. However, plasma cholesterol and PL concentrations were lower in fish fed the VO diets. Replacing FO with VO usually increases dietary phytosterol and reduces cholesterol content. Such dietary modifications have been reported to induce a decrease in plasma cholesterol and LDL-cholesterol both in humans ${ }^{(87,88)}$ and in fish ${ }^{(35-37,89)}$.

Phytosterols are structurally similar to cholesterol and may induce a relative cholesterol deficiency by mechanisms that interfere with cholesterol absorption such as competition for space in mixed micelles or competition with cholesterol transporters (such as ABC transporters) ${ }^{(87,90)}$. For instance, in Atlantic salmon, dietary inclusion of soyabean meal and soya saponins decreased the expression of $A B C G 5$ in the distal intestine $^{(91)}$ and in the liver ${ }^{(92)}$. In the present study, however, no repression of $A B C G 5$ expression related to diet composition was observed at hepatic or intestinal levels. Furthermore, no induction in the expression of the selected genes (CYP51A1 and $D H C R 7$ ) involved in the cholesterol biosynthesis was recorded in the intestine and liver of fish fed the VO diets. This differs from other studies in which hepatic or intestinal gene expression of key enzymes involved in cholesterol biosynthesis pathways (such as 3-hydroxy-3-methylglutaryl-CoA reductase, isopentenyl-diphosphate $\Delta$ isomerase, CYP51A1 or DHCR7) increased in response to the decreased plasma cholesterol concentration in fish fed plant-based ${ }^{(91-93)}$ or $\mathrm{VO}^{(7,10,39)}$ diets. Whether the lack of activation of cholesterol biosynthesis at the molecular level as a response to the reduced plasma cholesterol concentration that was observed in the present study is due to variations in the dietary cholesterol supply or due to species specific sensitivities to alternative ingredients, such as VO, remains to be clarified. 
(a)

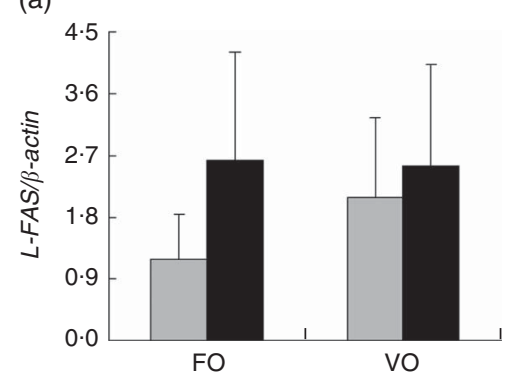

(b)

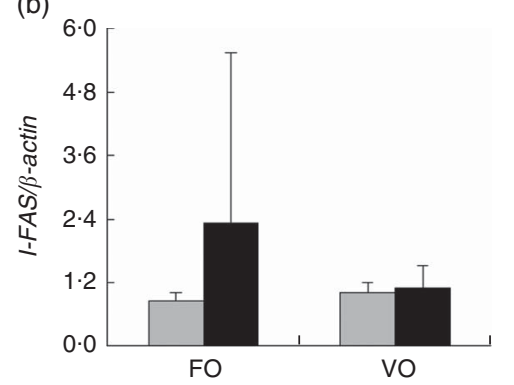

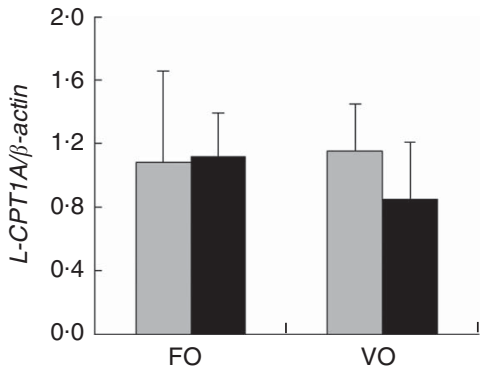
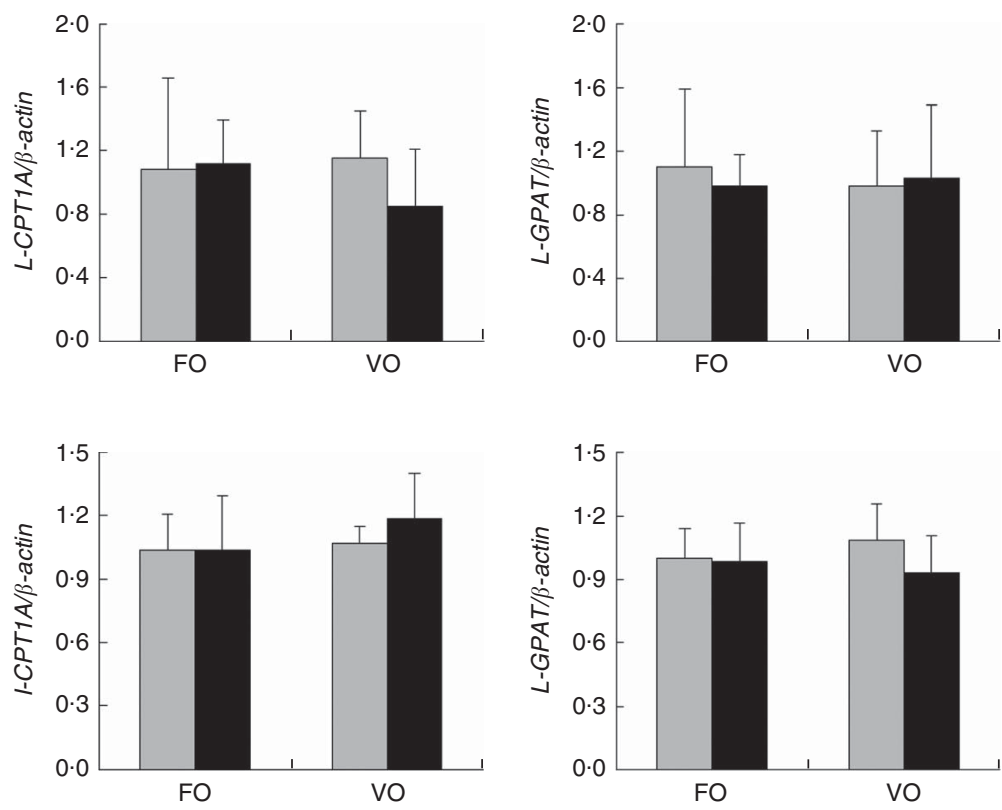

Fig. 2. mRNA levels of genes involved in lipogenesis (fatty acid synthase (FAS)), $\beta$-oxidation (carnitine palmitoyltransferase $1 A(C P T 1 A)$ ) and phospholipid synthesis (glycerol-3-phosphate acyltransferase (GPAT)) in the (a) liver and (b) intestine of gilthead sea bream fed the experimental diets. Expression values are normalised by $\beta$-actin-expressed transcripts. Fish oil (FO), blend of vegetable oils (VO); carbohydrate content: $0 \%(\square, \mathrm{CH}-)$ or $20 \%(\square, \mathrm{CH}+)$ gelatinised maize starch. Relative fold difference between treatments are presented as means $(n 6)$, with their standard deviations. Significant differences at $P<0.05$ (two-way ANOVA).
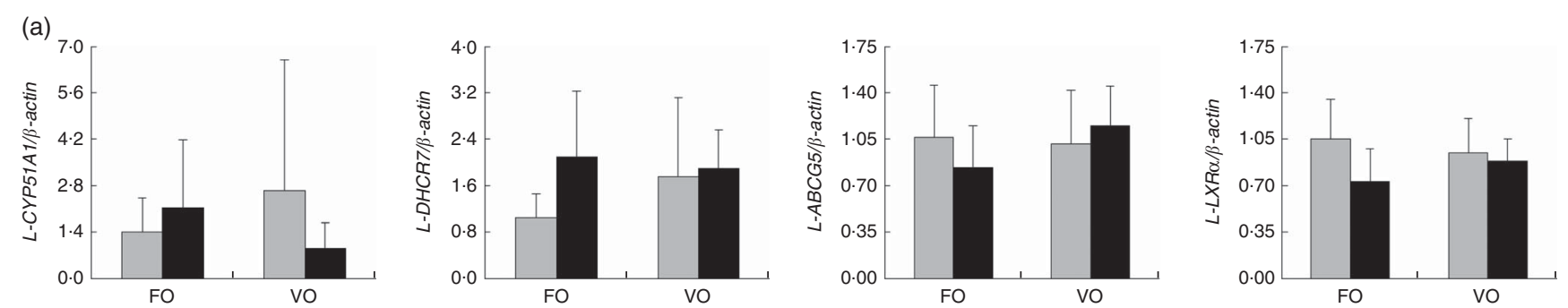

(b)
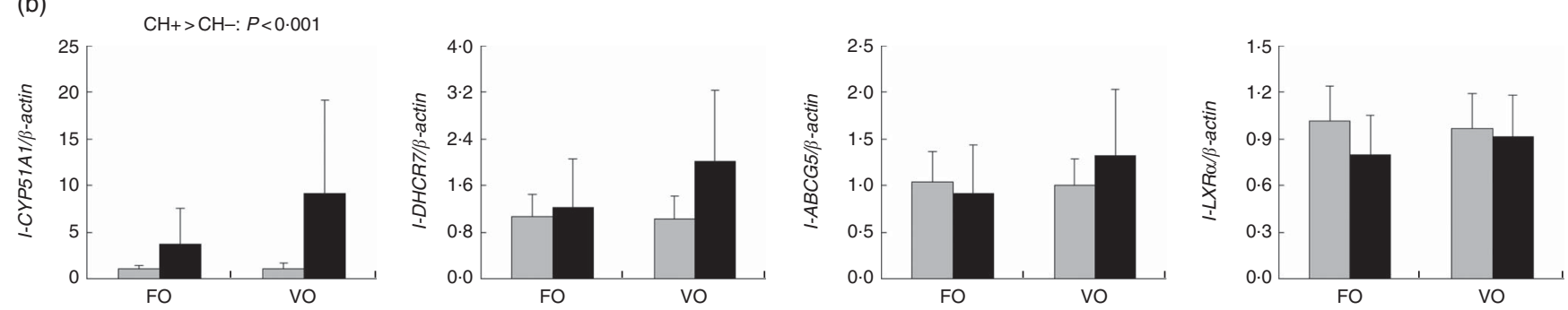

Fig. 3. mRNA levels of genes involved in cholesterol metabolism (lanosterol 14- $a$ demethylase, cytochrome P450 51 (CYP51A1); 7-dehydrocholesterol reductase (DHCR7); liver X receptor $a(\mathrm{LXR} a)$ ) and transport (ATP binding cassette G5 (ABCG5)) in the (a) liver and (b) intestine of gilthead sea bream fed the experimental diets. Expression values are normalised by $\beta$-actin-expressed transcripts. Fish oil (FO), blend of vegetable oils (VO); carbohydrate content: $0 \%(\square, \mathrm{CH}-)$ or $20 \%(\square, \mathrm{CH}+)$ gelatinised maize starch. Relative fold difference between treatments are presented as means $(n 6)$, with their standard deviations. Significant differences at $P<0.05$ (two-way ANOVA).

An intestinal and hepatic induction of expression or increased activity of enzymes involved in PL biosynthesis has been reported in carnivorous fish fed $\mathrm{VO}^{(38)}$ or plant feedstuffbased diets ${ }^{(93)}$. However, the biosynthesis rate seems to be insufficient to avoid an accumulation of lipid droplets in the enterocytes $^{(94,95)}$. This condition is usually associated with a PL deficit that promotes an impairment in lipoprotein assembly and export from enterocytes and reduces PL concentration in plasma $^{(7,35,36)}$.

In the present study, despite the lower plasma PL concentration in VO groups, a nutritional regulation of transcript levels of GPAT was not observed in the intestine or the in liver of gilthead sea bream fed the experimental diets. $G P A T$ is involved in the first steps of glycerol-3-phosphate 
(A)
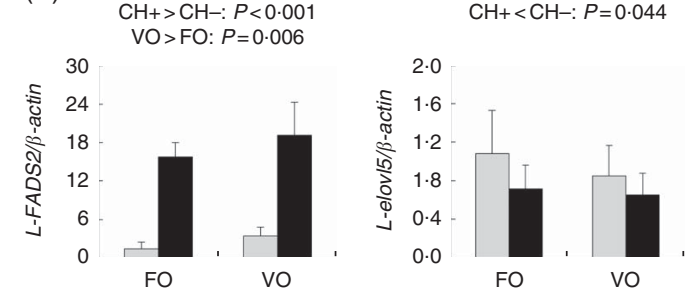

(B)
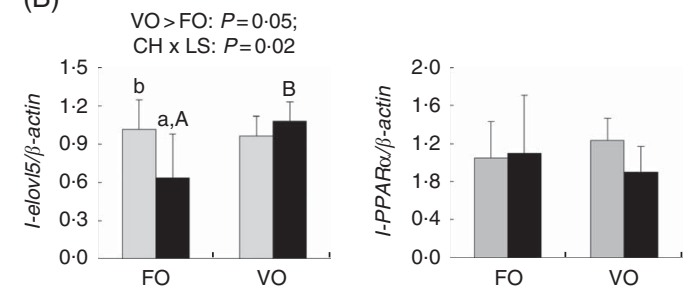
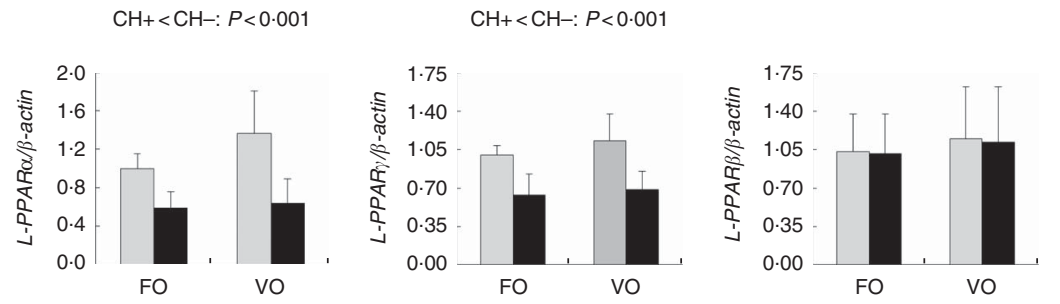

Fig. 4. mRNA levels of genes involved in the long-chain PUFA-biosynthesis pathway ( $\triangle 6$ fatty acyl desaturase (FADS2), elongase 5, (elovl5)) and transcription factors involved in several lipid-related processes $(P P A R a, \gamma, \beta)$ in the $(A)$ liver and $(B)$ intestine of gilthead sea bream fed the experimental diets. Expression values are normalised by $\beta$-actin-expressed transcripts. Fish oil (FO), blend of vegetable oils (VO); carbohydrate content: $0 \%$ ( $\square, \mathrm{CH}-$ ) or $20 \%$ (, $\mathrm{CH}+)$ gelatinised maize starch. Relative fold difference between treatments are presented as means $(n 6)$, with their standard deviations. Significant differences at $P<0.05$ (two-way ANOVA). If interaction was significant, one-way ANOVA was performed for each factor. ${ }^{a, b, A, B}$ Means with different capital and small letters indicate significant differences $(P<0.05)$ between the two tested lipid sources and two carbohydrate levels, respectively; means with no letters are not significantly different $(P>0.05)$.

pathway, which according to Caballero et al. ${ }^{(38)}$ is the main pathway implicated in intestinal PL synthesis in gilthead sea bream. Therefore, further investigation on the nutritional regulation of the enzymes involved in downstream steps of the PL biosynthesis pathways is required to understand PL biosynthesis regulation in gilthead sea bream.

Dietary lipid source is known to regulate lipogenesis and FA bioconversion pathways, which may affect tissue lipid deposition and FA composition. Although in some studies increased hepatic lipogenic enzymes activity and lipid content have been reported in fish fed diets in which dietary FO was replaced with $\mathrm{VO}^{(6,19)}$, in other studies such effects were not demonstrated $^{(7,35,36,96)}$. In the present study, dietary lipid source did not affect hepatic and muscular lipid content, and a decrease in whole-body lipid content was reported within $\mathrm{CH}$ - groups when FO was substituted by VO. On the other hand, liver and muscle FA composition was strongly influenced by dietary replacement of FO by VO, as previously reported in gilthead sea bream ${ }^{(97)}$ and in other species ${ }^{(7,8,29,98)}$. According to the predicted effects of dietary oils in muscle FA composition of 1-year-old gilthead sea bream with different nutritional backgrounds ${ }^{(97)}$, in the present study liver and muscle lipids increased the content of $18: 1 n-9,18: 2 n-6$ and $18: 3 n-3$, whereas total lipid reduced the content of $20: 5 n-3$ and $22: 6 n-3$ when FO was replaced by VO. Thus, despite the hepatic upregulation of FADS2 in fish fed the VO-based diets, the low proportion of LC-PUFA or their intermediary products such as $18: 3 n-6$ and $18: 4 n-3$ in VO groups suggest a limited FADS2 activity. Maximal FADS2 efficiency is thought to be modulated by the levels of substrate and product availability ${ }^{(30)}$. In this sense, in the present study the limited accumulation of FA intermediates could partly have been caused by an inadequate dietary supply of substrate and/or product availability. Accordingly, in a previous study with this species using a diet completely devoid of LC-PUFA and containing olive oil as the sole lipid source, Seiliez et al. ${ }^{(47)}$ observed higher transcript levels of FADS2 and increased accumulation of FA intermediates (18:2n-9, 20:2n-9 and 18:3n-6) in fish fed that diet comparatively to fish fed an LC-PUFA-rich diet, which suggested the existence of FADS2 activity.

In this study, the low efficacy in induction of FADS2 expression and of nutritional regulation of elovl5 transcripts by VO observed, along with lack of $\Delta 5$ desaturase activity observed in vitro ${ }^{(99)}$, may explain the low capacity of conversion of C18 PUFA into LC-PUFA at an appreciable rate in gilthead sea bream.

\section{Dietary carbohydrate content and lipid source interaction}

The interactions between dietary carbohydrate content and lipid source that were recorded on the whole-body, liver and plasma lipid content suggest that the overall effect of starch intake on lipid deposition was disturbed by the change in dietary lipid source. Indeed, an increase in lipid retention with dietary starch intake was more evident when fish were fed the VO-based diet. This assumption is supported by the higher, whole-body and liver lipid content in the $\mathrm{VOCH}+$ group than in the VOCH- group and can be related with the coupled increase in the lipogenic potential of dietary carbohydrates (higher liver FAS, ME and G6PD activities) and VO (higher liver G6PD activity). On the other hand, under an FO-based diet regimen, the unaltered lipid retention, liver and whole-body lipid content, but elevated TAG levels following starch intake, suggest a higher lipid mobilisation, transport and/or utilisation in the FOCH+ group than in the FOCH- group. No molecular markers for lipid uptake were assessed in this study in liver and intestine, and the one evaluated here and related to catabolism (CPT1A) does not help to understand or clarify this hypothesis 
because transcriptional regulation of these proteins by nutrients was not recorded.

At the same time, we also noticed that the stimulatory effect of dietary starch on muscle glycogen deposition was attenuated and became non-significant under the VO-based dietary regimen, probably related with the fact that dietary VO per se, but not in combination with carbohydrates, had an inductor effect on glycogenesis, as suggested by the higher muscle and liver glycogen levels in the $\mathrm{VOCH}$ - group than in the FOCH- group.

Furthermore, it was observed that intake of starch when coupled with dietary VO seemed to enhance the hypocholesterolemic effects of dietary $\mathrm{VO}$ as plasma cholesterol concentration decreased with carbohydrate intake only when fish were fed VO-based diet. It is important to note here that, within the molecular actors involved in cholesterol-related processes (CYP51A1, DHCR7, ABCG5, LXR $\alpha$ ) assessed in liver and intestine in the present study, no molecular difference induced by a nutritional interaction of nutrients that could reflect this phenotype was found. Therefore, the physiological or metabolic mechanisms underlying this finding remain to be demonstrated.

\section{Conclusion}

To our knowledge, this is the first study in a marine fish species reporting a transcriptional induction of FADS2 by dietary carbohydrate, in addition to VO. Although the $n$-3 LC-PUFA biosynthesis pathway was insufficient to compensate for the reduced dietary EPA and DHA in VO-based diets, this study provides new perspectives on the use of nutritional strategies for inducing LC-PUFA biosynthesis in marine fish species.

Furthermore, change in dietary lipid source seemed to modify the overall effect of starch intake on mechanisms involved in cholesterol body pools, lipid and glycogen body allocation. Considering that these metabolites greatly influence the fish quality, the present findings highlight the critical need to assess the potential effects between dietary nutrients on metabolic-related processes involved in tissue metabolites deposition, especially in the context of alternative aquafeeds rich in $\mathrm{VO}$ and carbohydrates.

\section{Acknowledgements}

The authors express their thanks to P. Correia and M. Cluzeaud for their technical assistance.

This work was partially supported by national funds through Foundation for Science and Technology (FCT) - under the project 'PEst-C/MAR/LA0015/2011' and co-financed by the European Regional Development Fund through the COMPETE Operational Competitiveness Programme. C. C. was supported by a grant (SFRH/BD/76297/2011) from FCT, Portugal, and A. D. was supported by the National Council of Technological and Scientific Development (CNPq), Brazil.

C. C. carried out the main experimental work and wrote the draft of the manuscript under the direction of the project designer and leaders A. O.-T., G. C. and S. P.; A. D. assisted with the biochemical analyses; and L. L. performed the fatty acid analyses. All authors contributed to and approved the manuscript.

The authors declare that there are no conflicts of interest.

\section{Supplementary material}

For supplementary material/s referred to in this article, please visit http://dx.doi.org/10.1017/S000711451600163X

\section{References}

1. Tocher DR (2015) Omega-3 long-chain polyunsaturated fatty acids and aquaculture in perspective. Aquaculture 449 , 94-107.

2. Food and Agriculture Organization (2014) The State of World Fisheries and Aquaculture 2014. Rome: Food and Agriculture Organization of the United Nations.

3. Enes P, Panserat S, Kaushik S, et al. (2009) Nutritional regulation of hepatic glucose metabolism in fish. Fish Physiol Biochem 35, 519-539.

4. Gatlin DM, Barrows FT, Brown P, et al. (2007) Expanding the utilization of sustainable plant products in aquafeeds: a review. Aquacult Res 38, 551-579.

5. Stone DAJ (2003) Dietary carbohydrate utilization by fish. Rev Fish Sci 11, 337-369.

6. Betancor M, Sprague M, Usher S, et al. (2015) A nutritionallyenhanced oil from transgenic Camelina sativa effectively replaces fish oil as a source of eicosapentaenoic acid for fish. Sci Rep 5, 8104.

7. Castro C, Corraze G, Pérez-Jiménez A, et al. (2015) Dietary carbohydrate and lipid source affect cholesterol metabolism of European sea bass (Dicentrarchus labrax) juveniles. BrJ Nutr 114, 1143-1156.

8. Jordal AEO, Lie O \& Torstensen BE (2007) Complete replacement of dietary fish oil with a vegetable oil blend affect liver lipid and plasma lipoprotein levels in Atlantic salmon (Salmo salar L.). Aquacult Nutr 13, 114-130.

9. Kjaer MA, Vegusdal A, Gjoen T, et al. (2008) Effect of rapeseed oil and dietary $n-3$ fatty acids on triacylglycerol synthesis and secretion in Atlantic salmon hepatocytes. Biochim Biophys Acta 1781, 112-122.

10. Leaver MJ, Villeneuve LA, Obach A, et al. (2008) Functional genomics reveals increases in cholesterol biosynthetic genes and highly unsaturated fatty acid biosynthesis after dietary substitution of fish oil with vegetable oils in Atlantic salmon (Salmo salar). BMC Genomics 9, 299.

11. Morais S, Edvardsen RB, Tocher DR, et al. (2012) Transcriptomic analyses of intestinal gene expression of juvenile Atlantic cod (Gadus morhua) fed diets with Camelina oil as replacement for fish oil. Comp Biochem Physiol B Biochem Mol Biol 161, 283-293.

12. Brauge C, Medale F \& Corraze G (1994) Effect of dietary carbohydrate levels on growth, body composition and glycaemia in rainbow trout, Oncorbynchus mykiss, reared in seawater. Aquaculture 123, 109-120.

13. Sheridan MA (1988) Lipid dynamics in fish: aspects of absorption, transportation and mobilization. Comp Biochem Physiol B 90, 679-690.

14. Tocher DR (2003) Metabolism and functions of lipids and fatty acids in teleost fish. Rev Fish Sci 11, 107-184.

15. Menoyo D, Izquierdo MS, Robaina L, et al. (2004) Adaptation of lipid metabolism, tissue composition and flesh quality in gilthead sea bream (Sparus aurata) to the replacement of 
dietary fish oil by linseed and soyabean oils. Br I Nutr 92 41-52.

16. Panserat S, Kolditz C, Richard N, et al. (2008) Hepatic gene expression profiles in juvenile rainbow trout (Oncorbynchus mykiss) fed fishmeal or fish oil-free diets. Br J Nutr 100, 953-967.

17. Morais S, Pratoomyot J, Taggart JB, et al. (2011) Genotypespecific responses in Atlantic salmon (Salmo salar) subject to dietary fish oil replacement by vegetable oil: a liver transcriptomic analysis. BMC Genomics 12, 255.

18. Morais S, Silva T, Cordeiro O, et al. (2012) Effects of genotype and dietary fish oil replacement with vegetable oil on the intestinal transcriptome and proteome of Atlantic salmon (Salmo salar). BMC Genomics 13, 448.

19. Peng $\mathrm{M}, \mathrm{Xu} \mathrm{W}$, Mai K, et al. (2014) Growth performance, lipid deposition and hepatic lipid metabolism related gene expression in juvenile turbot (Scophthalmus maximus L.) fed diets with various fish oil substitution levels by soybean oil. Aquaculture 433, 442-449.

20. Liang XF, Ogata HY \& Oku H (2002) Effect of dietary fatty acids on lipoprotein lipase gene expression in the liver and visceral adipose tissue of fed and starved red sea bream Pagrus major. Comp Biochem Physiol A 132, 913-919.

21. Stubhaug I, Froyland L \& Torstensen BE (2005) Beta-oxidation capacity of red and white muscle and liver in Atlantic salmon (Salmo salar L.) - effects of increasing dietary rapeseed oil and olive oil to replace capelin oil. Lipids 40, 39-47.

22. Jordal AEO, Torstensen BE, Tsoi S, et al. (2005) Dietary rapeseed oil affects the expression of genes involved in hepatic lipid metabolism in Atlantic salmon (Salmo salar L.). J Nutr 135, 2355-2361.

23. Turchini GM, Mentasti T, Froyland L, et al. (2003) Effects of alternative dietary lipid sources on performance, tissue chemical composition, mitochondrial fatty acid oxidation capabilities and sensory characteristics in brown trout (Salmo trutta L.). Aquaculture 225, 251-267.

24. Kamalam BS, Médale F, Kaushik S, et al. (2012) Regulation of metabolism by dietary carbohydrates in two lines of rainbow trout divergently selected for muscle fat content. J Exp Biol 215, 2567-2578.

25. Kamalam BS, Médale F, Larroquet L, et al. (2013) Metabolism and fatty acid profile in fat and lean rainbow trout lines fed with vegetable oil: effect of carbohydrates. PLOS ONE $\mathbf{8}$, e76570.

26. Jin J, Médale F, Kamalam BS, et al. (2014) Comparison of glucose and lipid metabolic gene expressions between fat and lean lines of rainbow trout after a glucose load. PLOS ONE 9 , e105548.

27. Seiliez I, Panserat S, Kaushik S, et al. (2001) Cloning, tissue distribution and nutritional regulation of a Delta 6-desaturaselike enzyme in rainbow trout. Comp Biochem Physiol B Biochem Mol Biol 130, 83-93.

28. González-Rovira A, Mourente G, Zheng XZ, et al. (2009) Molecular and functional characterization and expression analysis of a Delta 6 fatty acyl desaturase cDNA of European sea bass (Dicentrarchus labrax L.). Aquaculture 298, 90-100.

29. Tocher DR, Zheng X, Schlechtriem C, et al. (2006) Highly unsaturated fatty acid synthesis in marine fish: cloning, functional characterization, and nutritional regulation of fatty acyl $\Delta 6$ desaturase of Atlantic cod (Gadus morhua L.). Lipids 41, 1003-1016.

30. Vagner M \& Santigosa E (2011) Characterization and modulation of gene expression and enzymatic activity of delta- 6 desaturase in teleosts: a review. Aquaculture 315, 131-143.

31. Montero D \& Izquierdo M (2010) Welfare and health of fish fed vegetable oils as alternative lipid sources to fish oil. In Fish
Oil Replacement and Alternative Lipid Sources in Aquaculture Feeds, pp. 439-485 [GM Turchini, WK Ng and DR Tocher, editors]. Boca Raton, FL: CRC Press.

32. Torstensen B \& Tocher D (2010) The effects of fish oil replacement on lipid metabolism of fish. In Fish Oil Replacement and Alternative Lipid Sources in Aquaculture Feeds, pp. 405-437 [GM Turchini, WK Ng and DR Tocher, editors]. Boca Raton, FL: CRC Press.

33. NRC (2011) Nutrient Requirements of Fish and Shrimp. Washington, DC: The National Academies Press.

34. Tocher DR, Bendiksen EA, Campbell PJ, et al. (2008) The role of phospholipids in nutrition and metabolism of teleost fish. Aquaculture 280, 21-34.

35. Richard N, Kaushik S, Larroquet L, et al. (2006) Replacing dietary fish oil by vegetable oils has little effect on lipogenesis, lipid transport and tissue lipid uptake in rainbow trout (Oncorbynchus mykiss). Br J Nutr 96, 299-309.

36. Richard N, Mourente G, Kaushik S, et al. (2006) Replacement of a large portion of fish oil by vegetable oils does not affect lipogenesis, lipid transport and tissue lipid uptake in European seabass (Dicentrarchus labrax L.). Aquaculture 261, 1077-1087.

37. Morais S, Pratoomyot J, Torstensen BE, et al. (2011) Diet $\mathrm{x}$ genotype interactions in hepatic cholesterol and lipoprotein metabolism in Atlantic salmon (Salmo salar) in response to replacement of dietary fish oil with vegetable oil. Br J Nutr 106, 1457-1469.

38. Caballero MJ, Gallardo G, Robaina L, et al. (2006) Vegetable lipid sources affect in vitro biosynthesis of triacylglycerols and phospholipids in the intestine of sea bream (Sparus aurata). Br J Nutr 95, 448-454.

39. Liland NS, Espe M, Rosenlund G, et al. (2013) High levels of dietary phytosterols affect lipid metabolism and increase liver and plasma TAG in Atlantic salmon (Salmo salar L.). Br J Nutr 110, 1958-1967.

40. Agaba MK, Tocher DR, Zheng X, et al. (2005) Cloning and functional characterisation of polyunsaturated fatty acid elongases of marine and freshwater teleost fish. Comp Biochem Physiol B Biochem Mol Biol 142, 342-352.

41. Benedito-Palos L, Ballester-Lozano G \& Perez-Sanchez J (2014) Wide-gene expression analysis of lipid-relevant genes in nutritionally challenged gilthead sea bream (Sparus aurata). Gene 547, 34-42.

42. Benedito-Palos L, Calduch-Giner JA, Ballester-Lozano GF, et al. (2013) Effect of ration size on fillet fatty acid composition, phospholipid allostasis and mRNA expression patterns of lipid regulatory genes in gilthead sea bream (Sparus aurata). Br J Nutr 109, 1175-1187.

43. Calduch-Giner JA, Davey G, Saera-Vila A, et al. (2010) Use of microarray technology to assess the time course of liver stress response after confinement exposure in gilthead sea bream (Sparus aurata L.). BMC Genomics 11, 193.

44. Mininni AN, Milan M, Ferraresso S, et al. (2014) Liver transcriptome analysis in gilthead sea bream upon exposure to low temperature. BMC Genomics 15, 1-12.

45. Pérez-Sánchez J, Borrel M, Bermejo-Nogales A, et al. (2013) Dietary oils mediate cortisol kinetics and the hepatic mRNA expression profile of stress-responsive genes in gilthead sea bream (Sparus aurata) exposed to crowding stress. Implications on energy homeostasis and stress susceptibility. Comp Biochem Physiol D 8, 123-130.

46. Sánchez-Gurmaches J, Cruz-Garcia L, Ibarz A, et al. (2013) Insulin, IGF-I, and muscle MAPK pathway responses after sustained exercise and their contribution to growth and lipid metabolism regulation in gilthead sea bream. Domest Anim Endocrinol 45, 145-153. 
47. Seiliez I, Panserat S, Corraze G, et al. (2003) Cloning and nutritional regulation of a Delta 6-desaturase-like enzyme in the marine teleost gilthead seabream (Sparus aurata). Comp Biochem Physiol B Biochem Mol Biol 135, 449-460.

48. Castro C, Corraze G, Panserat S, et al. (2015) Effects of fish oil replacement by a vegetable oil blend on digestibility, postprandial serum metabolite profile, lipid and glucose metabolism of European sea bass (Dicentrarchus labrax) juveniles. Aquacult Nutr 21, 592-603.

49. Librán-Pérez M, Figueiredo-Silva AC, Panserat S, et al. (2013) Response of hepatic lipid and glucose metabolism to a mixture or single fatty acids: possible presence of fatty acid-sensing mechanisms. Comp Biochem Physiol A Mol Integr physiol 164, 241-248.

50. Menoyo D, Diez A, Lopez-Bote CJ, et al. (2006) Dietary fat type affects lipid metabolism in Atlantic salmon (Salmo salar L.) and differentially regulates glucose transporter GLUT4 expression in muscle. Aquaculture 261, 294-304.

51. Oliva-Teles A (2000) Recent advances in European sea bass and gilthead sea bream nutrition. Aquacult Int 8, 477-492.

52. AOAC (2012) Official Methods of Analysis of Association of Official Analytical Chemists International. Gaithersburg, MD: AOAC International.

53. Beutler HO (1984) Starch. In Methods of Enzymatic Analysis, pp. 2-10 [HU Bergmeyer, editor]. Basel: Verlag Chemie Weinheim.

54. Roehrig KL \& Allred JB (1974) Direct enzymatic procedure for the determination of liver glycogen. Anal Biochem 58, 414-421.

55. Folch J, Lees M \& Sloane Stanley GH (1957) A simple method for the isolation and purification of total lipides from animal tissues. J Biol Chem 226, 497-509.

56. Shantha N \& Ackman R (1990) Nervonic acid versus tricosanoic acid as internal standards in quantitative gas chromatographic analyses of fish oil longer-chain $n-3$ polyunsaturated fatty acid methyl esters. J Chromatogr B Biomed Sci Appl $\mathbf{5 3 3}$, $1-10$.

57. Stadtman TC (1957) Determination of cholesterol and ergosterol by Liebermann-Burchard reaction. Methods Enzymol 3, 362-365.

58. Rozen S \& Skaletsky HJ (2000) Primer3 on the WWW for general users and for biologist programmers. Methods Mol Biol 132, 365-386.

59. Enes P, Panserat S, Kaushik S, et al. (2008) Hepatic glucokinase and glucose-6-phosphatase responses to dietary glucose and starch in gilthead sea bream (Sparus aurata) juveniles reared at two temperatures. Comp Biochem Physiol A Mol Integr Physiol 149, 80-86.

60. Pfaffl MW (2001) A new mathematical model for relative quantification in real-time RT-PCR. Nucleic Acids Res 29, e45.

61. Couto A, Enes P, Peres H, et al. (2008) Effect of water temperature and dietary starch on growth and metabolic utilization of diets in gilthead sea bream (Sparus aurata) juveniles. Comp Biochem Physiol A Mol Integr Physiol 151, 45-50.

62. Enes P, Panserat S, Kaushik S, et al. (2008) Growth performance and metabolic utilization of diets with native and waxy maize starch by gilthead sea bream (Sparus aurata) juveniles. Aquaculture 274, 101-108.

63. Peres H, Gonçalves P \& Oliva-Teles A (1999) Glucose tolerance in gilthead seabream (Sparus aurata) and European seabass (Dicentrarchus labrax). Aquaculture 179, 415-423.

64. Engelking L (2010) Overview of carbohydrate metabolism. In Textbook of Veterinary Physiological Chemistry, 2nd ed. pp. 120-124 [L Engelking, editor]. Amsterdam: Academic Press.

65. Panserat S, Medale F, Blin C, et al. (2000) Hepatic glucokinase is induced by dietary carbohydrates in rainbow trout, gilthead seabream, and common carp. Am J Physiol Regul Integr Comp Physiol 278, R1164-R1170.

66. Panserat S, Plagnes-Juan E \& Kaushik S (2002) Gluconeogenic enzyme gene expression is decreased by dietary carbohydrates in common carp (Cyprinus carpio) and gilthead seabream (Sparus aurata). Biochim Biophys Acta 1579, 35-42.

67. Fernández F, Miquel AG, Córdoba M, et al. (2007) Effects of diets with distinct protein-to-carbohydrate ratios on nutrient digestibility, growth performance, body composition and liver intermediary enzyme activities in gilthead sea bream (Sparus aurata, L.) fingerlings. J Exp Mar Biol Ecol 343, 1-10.

68. Kamalam BS, Panserat S, Aguirre P, et al. (2013) Selection for high muscle fat in rainbow trout induces potentially higher chylomicron synthesis and PUFA biosynthesis in the intestine. Comp Biochem Physiol A Mol Integr Physiol 164, 417-427.

69. Polakof S, Álvarez R \& Soengas JL (2010) Gut glucose metabolism in rainbow trout: implications in glucose homeostasis and glucosensing capacity. Am J Physiol Regul Integr Comp Physiol 299, R19-R32.

70. Kortner TM, Björkhem I, Krasnov A, et al. (2014) Dietary cholesterol supplementation to a plant-based diet suppresses the complete pathway of cholesterol synthesis and induces bile acid production in Atlantic salmon (Salmo salar L.). Br J Nutr 111, 2089-2103.

71. Álvarez MJ, Lopez-Bote CJ, Diez A, et al. (1998) Dietary fish oil and digestible protein modify susceptibility to lipid peroxidation in the muscle of rainbow trout (Oncorbynchus mykiss) and sea bass (Dicentrarchus labrax). Br J Nutr 80, 281-289.

72. Viana Abranches M, Esteves de Oliveira FC \& Bressan J (2011) Peroxisome proliferator-activated receptor: effects on nutritional homeostasis, obesity and diabetes mellitus. Nutr Hosp 26, 271-279.

73. Leaver MJ, Bautista JM, Bjornsson BT, et al. (2008) Towards fish lipid nutrigenomics: current state and prospects for fin-fish aquaculture. Rev Fish Sci 16, 73-94.

74. Diez A, Menoyo D, Perez-Benavente S, et al. (2007) Conjugated linoleic acid affects lipid composition, metabolism, and gene expression in Gilthead sea bream (Sparus aurata L). J Nutr 137, 1363-1369.

75. Du ZY, Demizieux L, Degrace P, et al. (2004) Alteration of $20: 5 n-3$ and $22: 6 n-3$ fat contents and liver peroxisomal activities in fenofibrate-treated rainbow trout. Lipids 39 , 849-855.

76. Kennedy SR, Leavera MJ, Campbell PJ, et al. (2006) Influence of dietary oil content and conjugated linoleic acid (CLA) on lipid metabolism enzyme activities and gene expression in tissues of Atlantic salmon (Salmo salar L.). Lipids 41, 423-436.

77. Bonacic K, Estevez A, Bellot O, et al. (2016) Dietary fatty acid metabolism is affected more by lipid level than source in senegalese sole juveniles: interactions for optimal dietary formulation. Lipids 51, 105-122.

78. Zheng JL, Luo Z, Zhuo MQ, et al. (2014) Dietary L-carnitine supplementation increases lipid deposition in the liver and muscle of yellow catfish (Pelteobagrus fulvidraco) through changes in lipid metabolism. Br J Nutr 112, 698-708.

79. Dai W, Panserat S, Plagnes-Juan E, et al. (2015) Amino acids attenuate insulin action on gluconeogenesis and promote fatty acid biosynthesis via mTORC1 signaling pathway in trout hepatocytes. Cell Physiol Biochem 36, 1084-1100.

80. Ekmann KS, Dalsgaard J, Holm J, et al. (2013) Effects of dietary energy density and digestible protein:energy ratio on de novo lipid synthesis from dietary protein in gilthead sea bream (Sparus aurata) quantified with stable isotopes. Br J Nutr 110, 1771-1781.

81. Kirchner S, Kaushik S \& Panserat S (2003) Low protein intake is associated with reduced hepatic gluconeogenic enzyme 
expression in rainbow trout (Oncorbynchus mykiss). I Nutr 133, 2561-2564.

82. Lansard M, Panserat S, Plagnes-Juan E, et al. (2010) Integration of insulin and amino acid signals that regulate hepatic metabolism-related gene expression in rainbow trout: role of TOR. Amino Acids 39, 801-810.

83. Izquierdo MS, Montero D, Robaina L, et al. (2005) Alterations in fillet fatty acid profile and flesh quality in gilthead seabream (Sparus aurata) fed vegetable oils for a long terin period: recovery of fatty acid profiles by fish oil feeding. Aquaculture 250, 431-444.

84. Montero D, Mathlouthi F, Tort L, et al. (2010) Replacement of dietary fish oil by vegetable oils affects humoral immunity and expression of pro-inflammatory cytokines genes in gilthead sea bream Sparus aurata. Fish Shellfish Immunol 29, 1073-1081.

85. Benedito-Palos L, Saera-Vila A, Calduch-Giner JA, et al. (2007) Combined replacement of fish meal and oil in practical diets for fast growing juveniles of gilthead sea bream (Sparus aurata L.): networking of systemic and local components of GH/IGF axis. Aquaculture 267, 199-212.

86. Fountoulaki E, Vasilaki A, Hurtado R, et al. (2009) Fish oil substitution by vegetable oils in commercial diets for gilthead sea bream (Sparus aurata L.); effects on growth performance, flesh quality and fillet fatty acid profile: recovery of fatty acid profiles by a fish oil finishing diet under fluctuating water temperatures. Aquaculture 289, 317-326.

87. Brufau G, Canela MA \& Rafecas M (2008) Phytosterols: physiologic and metabolic aspects related to cholesterollowering properties. Nutr Res 28, 217-225.

88. MacKay DS \& Jones PJH (2011) Phytosterols in human nutrition: type, formulation, delivery, and physiological function. Eur J Lipid Sci Tech 113, 1427-1432.

89. Gilman CI, Leusch FD, Breckenridge WC, et al. (2003) Effects of a phytosterol mixture on male fish plasma lipoprotein fractions and testis $\mathrm{P} 450 \mathrm{scc}$ activity. Gen Comp Endocrinol 130, 172-184.

90. Ostlund RE Jr (2002) Phytosterols in human nutrition. Annu Rev Nutr 22, 533-549.

91. Kortner TM, Gu J, Krogdahl A, et al. (2013) Transcriptional regulation of cholesterol and bile acid metabolism after dietary soyabean meal treatment in Atlantic salmon (Salmo salar L.). Br J Nutr 109, 593-604.

92. Gu M, Kortner TM, Penn M, et al. (2014) Effects of dietary plant meal and soya-saponin supplementation on intestinal and hepatic lipid droplet accumulation and lipoprotein and sterol metabolism in Atlantic salmon (Salmo salar L.). Br J Nutr 111, 432-444.

93. Geay F, Ferraresso S, Zambonino-Infante JL, et al. (2011) Effects of the total replacement of fish-based diet with plant-based diet on the hepatic transcriptome of two European sea bass (Dicentrarchus labrax) half-sibfamilies showing different growth rates with the plant-based diet. BMC Genomics 12, 522 .

94. Caballero MJ, Izquierdo MS, Kjorsvik E, et al. (2003) Morphological aspects of intestinal cells from gilthead seabream (Sparus aurata) fed diets containing different lipid sources. Aquaculture 225, 325-340.

95. Olsen RE, Dragnes BT, Myklebust R, et al. (2003) Effect of soybean oil and soybean lecithin on intestinal lipid composition and lipid droplet accumulation of rainbow trout, Oncorbynchus mykiss walbaum. Fish Physiol Biochem 29, 181-192.

96. Torstensen BE, Froyland L \& Lie O (2004) Replacing dietary fish oil with increasing levels of rapeseed oil and olive oil - effects on Atlantic salmon (Salmo salar L.) tissue and lipoprotein lipid composition and lipogenic enzyme activities. Aquacult Nutr 10, 175-192.

97. Benedito-Palos L, Bermejo-Nogales A, Karampatos AI, et al. (2011) Modelling the predictable effects of dietary lipid sources on the fillet fatty acid composition of one-year-old gilthead sea bream (Sparus aurata L.). Food Chem 124, $538-544$

98. Benitez-Dorta V, Caballero MJ, Izquierdo M, et al. (2013) Total substitution of fish oil by vegetable oils in senegalese sole (Solea senegalensis) diets: effects on fish performance, biochemical composition, and expression of some glucocorticoid receptor-related genes. Fish Physiol Biochem 39, 335-349.

99. Tocher DR \& Ghioni C (1999) Fatty acid metabolism in marine fish: low activity of fatty acyl Delta 5 desaturation in gilthead sea bream (Sparus aurata) cells. Lipids 34, 433-440. 